This item was submitted to Loughborough's Research Repository by the author.

Items in Figshare are protected by copyright, with all rights reserved, unless otherwise indicated.

\title{
Brittle interfacial cracking between two dissimilar elastic layers: part 2- numerical verification
}

PLEASE CITE THE PUBLISHED VERSION

http://dx.doi.org/10.1016/j.compstruct.2015.06.079

PUBLISHER

(c) Elsevier

VERSION

AM (Accepted Manuscript)

\section{PUBLISHER STATEMENT}

This work is made available according to the conditions of the Creative Commons Attribution-NonCommercialNoDerivatives 4.0 International (CC BY-NC-ND 4.0) licence. Full details of this licence are available at: https://creativecommons.org/licenses/by-nc-nd/4.0/

\section{LICENCE}

CC BY-NC-ND 4.0

\section{REPOSITORY RECORD}

Harvey, Christopher M., Joseph D. Wood, and Simon Wang. 2015. "Brittle Interfacial Cracking Between Two Dissimilar Elastic Layers: Part 2-numerical Verification”. figshare. https://hdl.handle.net/2134/18652. 


\title{
Brittle interfacial cracking between two dissimilar elastic layers: Part 2-Numerical verification
}

\author{
C. M. Harvey, J. D. Wood, S. Wang* \\ Department of Aeronautical and Automotive Engineering, Loughborough University, \\ Loughborough, Leicestershire LE11 3TU, United Kingdom
}

\begin{abstract}
A thorough program of 2D finite element method (FEM) simulations is carried out parametrically on a bimaterial double cantilever beam (DCB) model in MSC/NASTRAN. The Young's modulus ratio, the Poisson's ratio, the thickness ratio, and the DCB tip loads are varied over their entire practically useful domains for different values of the crack extension size. Extensive comparisons are made between the results of the analytical theory that was developed in Part 1 by Harvey et al. (2015) and FEM results. This paper reports the outcome of these comparisons. The present analytical theory and the supporting mathematical techniques are thoroughly verified. Overall, excellent agreement is observed between the present analytical theory and the FEM results for the crack extension size-dependent energy release rate (ERR) components and the stress intensity factors (SIFs).

Keywords: Bimaterials, Energy release rate, Interfacial fracture, Stress intensity factors, Mixedmode partition, Orthogonal pure modes
\end{abstract}

\footnotetext{
* Corresponding Author
} 


\begin{tabular}{|c|c|}
\hline \multicolumn{2}{|l|}{ Nomenclature } \\
\hline$E_{1}, E_{2}$ & Young's modulus of upper and lower beams \\
\hline$G, G_{I}, G_{I I}$ & total, mode I and mode II ERRs \\
\hline$h_{1}, h_{2}, h$ & thicknesses of upper, lower and intact beams \\
\hline$K_{I}, K_{I I}$ & real and imaginary parts of the complex stress intensity factor \\
\hline$M_{1}, M_{2}$ & DCB tip bending moments on upper and lower beams \\
\hline$M_{1 B}, M_{2 B}, M_{B}$ & crack tip bending moments on upper, lower and intact beams \\
\hline$N_{1}, N_{2}$ & DCB tip axial forces on upper and lower beams \\
\hline$N_{1 B}, N_{2 B}, N_{B}$ & crack tip axial forces on upper, lower and intact beams \\
\hline$\beta_{i}, \beta_{i}^{\prime}$ & load-type pure mode II modes (with $i=1,2,3$ ) \\
\hline$\tilde{\beta}_{K}, \tilde{\theta}_{K}$ & approximate SIF-type pure mode II and pure mode I modes \\
\hline$\gamma$ & thickness ratio, $\gamma=h_{2} / h_{1}$ \\
\hline$\delta a$ & crack extension size \\
\hline$\eta$ & Young's modulus ratio, $\eta=E_{2} / E_{1}$ \\
\hline$\theta_{i}, \theta_{i}^{\prime}$ & load-type pure mode I modes (with $i=1,2,3$ ) \\
\hline$\mu_{1}, \mu_{2}$ & shear modulus of upper and lower beams \\
\hline$v_{1}, v_{2}$ & Poisson's ratio of upper and lower beams \\
\hline DCB & double cantilever beam \\
\hline ERR & energy release rate \\
\hline FEM & finite element method \\
\hline SIF & stress intensity factor \\
\hline VCCT & virtual crack closure technique \\
\hline
\end{tabular}




\section{Introduction}

Since Williams [1] discovered the complex stress intensity factor (SIF), $K=K_{I}+i K_{I I}$, for brittle interfacial cracking between two dissimilar elastic layers in 1959, it has been one of the most challenging fracture mechanics problems to develop an analytical theory to accurately calculate $K_{I}$ and $K_{I I}$, and the crack extension size-dependent energy release rate (ERR) components, $G_{I}$ and $G_{I I}$. In Part 1 of this work [2], Harvey et al. have developed such an analytical theory to accurately calculate $K_{I}, K_{I I}, G_{I}$ and $G_{I I}$ for brittle interfacial cracking between two dissimilar elastic layers in the double cantilever beam (DCB) shown in Fig. 1. This DCB is a representative mechanical model for many practical situations. The development is based on a fundamental mechanical understanding of the problem, a novel and powerful methodology and the previous work in Refs. [3-14]. This paper reports a thorough verification of the present analytical theory by using numerical results from finite element method (FEM) simulations. The FEM simulation procedure is described in Section 2. Extensive comparisons are made in Section 3 between the present analytical theory [2] and the FEM data. Conclusions are made in Section 4.

\section{FEM simulation procedure}

To verify the present analytical theory and the supporting mathematical techniques that were developed in Part 1 of this work [2] for brittle interfacial cracking between two dissimilar elastic layers, a thorough program of 2D FEM simulations was carried out parametrically on the DCB shown in Fig. 1 using MSC/NASTRAN. The Young's modulus ratio $\eta=E_{2} / E_{1}$ was varied in the range $1 / 100 \leq \eta \leq 100$; the Poisson's ratio $v_{1}=v_{2}=v$ was varied in the range $0 \leq v<0.5$;

the thickness ratio $\gamma=h_{2} / h_{1}$ was varied in the range $1 / 10 \leq \gamma \leq 10$; and the DCB tip loads, $M_{2}$, $N_{1}$ and $N_{2}$, were varied in the range $-20000 \leq M_{2}, N_{1}, N_{2} \leq 20000$ with $M_{1}=1000$. In this way the entire practically useful domain of cracking between bimaterial layers was considered. Since the FEM is dimensionless in nature, the model's parameters are given here without units. However, if engineering scale-appropriate units are desired in the following, then units of mm and $\mathrm{N}$ may be chosen for length and force respectively, from which the consistent set of units follows.

The top and bottom layers in the DCB were modelled using quadrilateral plane-strain shell elements with a thickness of $b=10$ and isotropic material properties within each layer. The 
minimum Young's modulus $E_{\min }=1000$. The Young's modulus of the top and bottom layers therefore varied with $E_{1}=E_{\min }$ and $E_{2}=\eta E_{\min }$ respectively if $\eta>1$, and with $E_{1}=E_{\min } / \eta$ and $E_{2}=E_{\text {min }}$ if $\eta<1$. The shear modulus was calculated as $\mu=E /[2(1+v)]$. The minimum beam thickness $h_{\min }=1$. The thickness of the top and bottom layers therefore varied with $h_{1}=h_{\min }$ and $h_{2}=h_{\min }$ respectively if $\gamma>1$, and with $h_{1}=h_{\min } / \gamma$ and $h_{2}=h_{\min }$ if $\gamma<1$. The uncracked length of the DCB $L=100$ and the cracked length $a=10$. The interface between the top and bottom layers was modelled with normal and shear point springs with a stiffness of $k_{s}=10^{11}$, which was sufficiently high in comparison to $E_{1}$ and $E_{2}$ to simulate brittle interfacial cracking without introducing excessive numerical error. Contact between the upper and lower surfaces of the crack was not considered.

Generally, the desired crack extension size $\delta a$ (over which the ERR was calculated) determined the size of the elements surrounding the crack tip, rather than the requirement for mesh independence. Since $\delta a$ is typically a small quantity ( $\delta a<<a$ ), non-uniform meshes were used in order to avoid excessive computation. Up to 2000 square elements of size $p \times p$ were centered on the crack tip in the $x$-direction, and up to 100 square elements were centered on the crack tip in the $y$-direction. Unless noted otherwise, in this paper $p=0.01$ is used. The crack extension size $\delta a$ is specified for each set of simulations in Section 3. Beyond the region of uniform element size surrounding the crack tip, elements were allowed to grow at a constant rate of 1.1 in both the $x$ - and $y$-directions up to a maximum size of 1.0, after which they remained at this maximum size. Very small adjustments were made to the element size growth rate where necessary to satisfy the boundary geometry. Axial forces, $N_{1}$ and $N_{2}$, were applied as point forces to the ends of the top and bottom layers respectively and were uniformly-distributed by area. Bending moments, $M_{1}$ and $M_{2}$, were applied as equal and opposite axial forces in the topand bottom-right corners of the top and bottom layers respectively. Because the interface is rigid, the ERRs were calculated using the virtual crack closure technique (VCCT) and interfacial point springs $[15,16,17]$ and as many spring pairs as exist inside the specified crack extension size $\delta a$. For example, if the element size at the crack tip is $p=0.01$ and the ERRs are to be calculated for a crack extension size of $\delta a=0.05$, then five normal and shear springs must be used in the VCCT calculation for $G_{I}$ and $G_{I I}$ respectively. 


\section{Numerical verification}

\subsection{The shifting technique}

The shifting technique is based on the observations that when $\delta a=0.05$ and $M_{2 B} / M_{1 B}=0$, (1) the ERR partitions from FEM simulations with $\gamma=1$ and $v=0.29$ are very close to the results from the authors' Timoshenko beam partition theory $[4,6,7,9]$ for the entire range of the modulus ratio $1 / 100 \leq \eta \leq 100$ with $\gamma=1$; and (2) the FEM partition results for the entire ranges of $1 / 10 \leq \gamma \leq 10,0 \leq v \leq 0.5$ and $1 / 100 \leq \eta \leq 100$ correspond to a non-uniform shift along the $G_{I} / G$-axis of the Timoshenko beam partition results with $\gamma=1$. Part 1 of this work [2] proposes a form for the shift based on $\gamma \leq 1$ and gives the empirical coefficients.

Fig. 2 confirms that the shifting technique accurately predicts the ERR partitions from FEM simulations with $\delta a=0.05, M_{2 B}=0$ and $M_{1 B}=1000$ and with $v=0.29$. In the figure, there is a marker and a line for each value of $\gamma$. The marker indicates the value from the FEM simulation, and the line indicates the analytical value, which is based on the shifting technique. Results are only shown for $\gamma \leq 1$ since the shift has only been determined for this region. There is excellent agreement between the value of $G_{I} / G$ determined from the shifting technique and that determined from the FEM for all values of $\gamma$ and $\eta$. The shifting technique will be further tested in Section 3.2 with different values of the Poisson's ratio $v$.

\subsection{Calculating the complete set of orthogonal pure modes}

The shifting technique, verified in Section 3.1, allows pure mode I $\theta_{1}$ mode to be determined directly for given values of $\gamma, \eta$ and $v$. It is then easy to determine the complete set of pure modes by making use of the orthogonality condition. If the values of $\theta_{1}$ and its orthogonal pure modes, $\beta_{1}, \theta_{2}, \beta_{2}, \theta_{3}$ and $\beta_{3}$, are correct, then $G_{I}$ and $G_{I I}$ can be calculated analytically for any combination of $M_{1 B}, M_{2 B}, N_{1 B}$ and $N_{2 B}$ with $\delta a=0.05$, and they will agree well with those values calculated from FEM simulations. If the pure modes are not accurately calculated, then it will not be possible to get agreement between the present analytical theory and the FEM for other loading conditions besides $M_{2 B} / M_{1 B}=0$. The purpose of this section is to assess the accuracy of the pure modes, $\theta_{1}, \beta_{1}, \theta_{2}, \beta_{2}, \theta_{3}$ and $\beta_{3}$. Note that no SIFs are involved in this section. 
Fig. 3 compares $G_{I} / G$ from the present analytical theory (lines) with that from the FEM (markers) for $M_{2 B}=-1000$ and $M_{1 B}=1000$ with $\delta a=0.05$ and $v=0.29$ for different values of $\gamma$ and $\eta$. For cases where $\gamma>1, \theta_{1}$ and $\beta_{1}$ are determined by making use of physical symmetry. Fig. 3 shows excellent agreement between the present analytical theory and the FEM data. The small 'dips' in the present analytical theory at $\log _{10}(1 / \gamma)= \pm 1$ and $\log _{10}(1 / \eta) \approx \mp 1.5$ can be explained by considering Fig. 2. When $\log _{10}(1 / \gamma)=1$ and $\log _{10}(1 / \eta) \approx-1.5$, the ERR partition $G_{I} / G$ becomes close to zero and therefore $\beta_{1}$ also becomes close to zero. Any numerical inaccuracies in the empirical shifting technique, from which $\theta_{1}$ and the orthogonal $\beta_{1}$ are calculated, therefore become magnified and this makes it more difficult to calculate $\theta_{1}$ and $\beta_{1}$ with the same degree of accuracy. Furthermore, recall from Part 1 of this work [2] that when $\delta a=0.05$ then $\theta_{1}^{\prime} \approx \theta_{1}$ and $\beta_{1}^{\prime} \approx \beta_{1}$. Because $\theta_{1}$ and $\theta_{1}^{\prime}$ do not coincide exactly when $\delta a=0.05$ (and neither do $\beta_{1}$ and $\beta_{1}^{\prime}$ ), it is possible for $G_{I} / G$ to become just less than 0 , or just greater than 1 . The present analytical theory cannot allow this, so the amount of shift is capped in some cases to prevent this from happening. For $\log _{10}(1 / \gamma)=1$, capping is employed when $\log _{10}(1 / \eta) \leq-1.5$. The effect of this is the small dip in the analytical theory in Fig. 3 at $\log _{10}(1 / \gamma)=1$ and $\log _{10}(1 / \eta)=-1.5$, and also the small dip at $\log _{10}(1 / \gamma)=-1$ and $\log _{10}(1 / \eta)=1.5$ due to physical symmetry.

Fig. 4a shows the effect of Poisson's ratio on the shifting technique and Fig. 4b shows its ability to determine the pure modes. The figure shows that the shifting technique works extremely well for $v \geq 0.29$, however, for smaller values of $v$, the adverse effect of capping on the shift increases for the same ranges of $\gamma$ and $\eta$ noted above and becomes severe for very small values of $v$. As $v$ increases above 0.3 , the capping rapidly disappears entirely and this explains the excellent performance in this region. Overall, it is concluded that the shifting technique works very well and that $\theta_{1}$ and $\beta_{1}$ can be calculated with high accuracy when $\delta a=0.05$ over the entire ranges of $1 / 100 \leq \eta \leq 100,0 \leq v<0.5$ and $1 / 10 \leq \gamma \leq 10$, except for the small 'corner' locations which represent very thin stiff layer spalling. Therefore to avoid repetitive verification in the following tests, a fixed Poisson's ratio of $v=0.29$ is used, which is slightly lower than the common value in many engineering cases of $v=1 / 3$ [18]. When $v=1 / 3$, it is expected that at least equal accuracy to when $v=0.29$ will be achieved since the capping effect disappears, as shown in Fig. 4. 
Figs. 5 and 6 assess the accuracy of the $\theta_{2}$ and $\theta_{3}$ values respectively (and the orthogonal values of $\beta_{2}$ and $\beta_{3}$ ), obtained from the use of the shifting technique and the orthogonality condition. The loading case in Fig. 5 is $N_{1 B}=10000$ and $M_{1 B}=1000$ with $M_{2 B}=N_{2 B}=0$, and the loading case in Fig. 6 is $N_{2 B}=10000$ and $M_{1 B}=1000$ with $M_{2 B}=N_{1 B}=0$. The agreement between the present analytical theory and the FEM is excellent everywhere, but slightly less good for the same values of $\gamma$ and $\eta$ noted above for Fig. 3. Overall, it is concluded that $\theta_{2}, \beta_{2}, \theta_{3}$ and $\beta_{3}$ can also be calculated with high accuracy when $\delta a=0.05$ by using the shifting technique and the orthogonality condition.

\subsection{Calculating and choosing the stress intensity factors}

Section 3.2 confirms that the ERR components, $G_{I}$ and $G_{I I}$, can be accurately determined for any combination of $M_{1 B}, M_{2 B}, N_{1 B}$ and $N_{2 B}$ with $\delta a=0.05$ and for any given values of $\gamma$ and $\eta$. Sun and Qian [13] and Part 1 of this work [2] have shown that for given values of $G_{I}$ and $G_{I I}$, there are four pairs of solutions for $K_{I}$ and $K_{I I}$, of which only one is mechanically admissible. In Ref. [13], FEM simulations were used to determine the correct pair. In Part 1 of this work [2], a method was devised to guide the selection of the correct pair by purely analytical means, in which an approximate pair of SIFs, $K_{I}$ and $K_{I I}$, is calculated by partitioning the total

ERR $G$ using approximate SIF-based orthogonal pure modes, $\tilde{\theta}_{K}$ and $\widetilde{\beta}_{K}$. The purpose of this section is to verify that (1) the approximate pair of SIFs can correctly and robustly guide the choice of the one physically correct accurate pair of SIFs from among the four that are provided mathematically, and that (2) the chosen SIFs are accurate.

Figs. 7 and 8 compare $K_{I}$ and $K_{I I}$ respectively for $M_{2 B}=0$ and $M_{1 B}=1000$ with $\delta a=0.05$ for different values of $\gamma$ and $\eta$, as obtained from the FEM (markers), the present analytical theory (lines), and the approximate analytical method (lines with small markers). Note that these figures are counterparts to Fig. 2. The FEM and the present analytical theory each provide $G_{I}$ and $G_{I I}$ for $\delta a=0.05$ (as in Section 3.2). The SIFs are then calculated by using the relationships between $G_{I}, G_{I I}$ and $K_{I}, K_{I I}$ [2,13]. In both cases, only the pair of SIFs that is closest to the approximate pair of SIFs is shown. For all values of $\gamma$ and $\eta$, excellent agreement is observed between the SIFs from the present analytical theory and those from the FEM. Furthermore, very close agreement is also observed between these SIFs and the approximate ones. Small 
discrepancies are seen, but this is of course expected due to it being an approximate method. The important observation is that the approximate SIFs are close enough to one of the four mathematical pairs of SIFs to accurately guide the correct choice.

Figs. 9 and 10, as the counterpart to Fig. 3, compare $K_{I}$ and $K_{I I}$ respectively for $M_{2 B}=-1000$ and $M_{1 B}=1000$ with $\delta a=0.05$ for different values of $\gamma$ and $\eta$. Figs. 11 and 12, as the counterpart to Fig. 5, compare $K_{I}$ and $K_{I I}$ respectively for $N_{1 B}=10000$ and $M_{1 B}=1000$ with $\delta a=0.05$. Figs. 13 and 14 , as the counterpart to Fig. 6, compare $K_{I}$ and $K_{I I}$ respectively for $N_{2 B}=10000$ and $M_{1 B}=1000$ with $\delta a=0.05$. In all these figures, markers represent data obtained from the FEM, lines represent the present analytical theory, and lines with small markers represent the approximate analytical method. As before, (1) excellent agreement is observed between the SIFs from the present analytical theory and those from the FEM, and (2) very close agreement is also observed between these SIFs and the approximate ones.

To further verify that the approximate SIFs can guide the choice of the correct pair of SIFs and to confirm the accuracy of the chosen SIFs, Fig. 15 compares the ERR partitions $G_{I} / G$ from the present analytical theory (lines) and from the FEM (markers) at a different crack extension size of $\delta a=0.01$. The analytical $G_{I} / G$ is calculated using the relationships between $G_{I}, G_{I I}$

and $K_{I}, K_{I I}$ [2,13]. In the present analytical theory [2], $K_{I}$ and $K_{I I}$ are calculated at a crack extension size of $\delta a=0.05$, that is, by using the ERRs, $G_{I}$ and $G_{I I}$, at $\delta a=0.05$. If they are correctly chosen and accurately calculated, the analytical $G_{I} / G$ at $\delta a=0.01$ should be in an excellent agreement with the $G_{I} / G$ from FEM simulations at $\delta a=0.01$. Fig. 15 shows that this is indeed the case. Note that the effect of capping is again evident at $\log _{10}(1 / \gamma)= \pm 1$ and $\log _{10}(1 / \eta) \approx \mp 1.5$.

Overall, it can be concluded that the present analytical theory can robustly choose the physically correct pair of SIFs from among the four that are provided mathematically. Also the present analytical theory accurately calculates the SIFs. Excellent agreement is seen between the present analytical theory and the FEM data for almost the entire domain of $\gamma$ and $\eta$.

\subsection{Calculating the energy release rate partitions}

So far in this numerical verification, Sections 3.1 to 3.3 combined have shown that the present analytical theory can accurately calculate the SIFs, $K_{I}$ and $K_{I I}$, for any combination of $M_{1 B}$, $M_{2 B}, N_{1 B}$ and $N_{2 B}$, and for any given values of $\gamma$ and $\eta$. To reinforce this verification, more 
extensive comparisons between the ERR partitions $G_{I} / G$ from the present analytical theory and from the FEM are presented in this section, with the aim of showing that the present analytical theory accurately determines the crack size-dependent ERRs, $G_{I}$ and $G_{I I}$.

Figs. 16 and 17 show the difference between the values of $G_{I} / G$ obtained from 2D FEM simulations and the values predicted by the present analytical theory over the entire practically useful domain of cracking between bimaterial layers. The Young's modulus ratio $\eta$ was varied in the range $1 / 100 \leq \eta \leq 100$; the thickness ratio $\gamma$ was varied in the range $1 / 10 \leq \gamma \leq 10$; and the DCB tip loads, $M_{2}, N_{1}$ and $N_{2}$, were varied in the range $-20000 \leq M_{2}, N_{1}, N_{2} \leq 20000$ with $M_{1}=1000$. Fig. 16 considers the variation of the crack tip bending moment ratio, $M_{2 B} / M_{1 B}$ with $M_{1 B}=1000$ at crack extension sizes of $\delta a=0.01$ and $\delta a=0.1$. Note that the sizes of the elements at the crack tip are $p=0.01$ and $p=0.1$ respectively. Fig. 17 considers the variation of $N_{1 B} / M_{1 B}$ and $N_{2 B} / M_{1 B}$ with $M_{1 B}=1000$ and $\delta a=p=0.1$. In all cases in Figs. 16 and 17, if $1 / 10 \leq \eta \leq 10$ then the maximum difference between $G_{I} / G$ from the present analytical theory and $G_{I} / G$ from the 2D FEM is 0.03 across the whole range of $\gamma$ and all the loading conditions. This is extremely close agreement. Outside of this range of $\eta$, the same level of agreement is mainly achieved except for a very small 'corner' location in some loading conditions. The reasons for this are the same as those given above for Figs. 3 to 6 in Section 3.2.

Overall, it can be concluded that the present analytical theory is able to calculate the ERR partitions, $G_{I}$ and $G_{I I}$, to a very high level of accuracy in relation to the FEM over the entire practically useful domain of cracking between bimaterial layers.

\section{Conclusions}

By carrying out a thorough program of parametric 2D FEM simulations on a bimaterial DCB model in MSC/NASTRAN, the following discoveries and conclusions have been made:

(1) The shifting technique, developed in Part 1 of this work [2], which has been developed for $\gamma \leq 1$, is able to accurately give the ERR partition $G_{I} / G$ when $\delta a=0.05$ and $M_{2 B} / M_{1 B}=0$.

(2) The complete set of orthogonal pure modes, $\theta_{1}, \beta_{1}, \theta_{2}, \beta_{2}, \theta_{3}$ and $\beta_{3}$, can be calculated with high accuracy when $\delta a=0.05$ by using the shifting technique and the orthogonality relationship that exists between them. This allows the present analytical theory to accurately calculate the ERR partition $G_{I} / G$ for any combination of crack tip bending moments and axial forces and any given values of $\gamma, \eta$ and $v$, when $\delta a=0.05$. 
(3) The present analytical theory [2] accurately calculates the SIFs. The approximate SIFs, which are calculated by partitioning the total ERR $G$ using approximate SIF-based orthogonal pure modes, $\tilde{\theta}_{K}$ and $\tilde{\beta}_{K}$, are in excellent agreement with the numerically accurate ones. Furthermore, the approximate pair of SIFs are sufficiently close to the one physically correct pair of SIFs to allow the correct pair to be chosen from among the four that are provided mathematically.

(4) Conclusions (1) to (3) combined allow the ERR components, $G_{I}$ and $G_{I I}$ to be calculated for any crack extension size $\delta a$ with a very high level of accuracy over the entire practically useful domain of cracking between bimaterial layers.

(5) The present analytical theory and the supporting mathematical techniques that were developed in Part 1 of this work [2] for brittle interfacial cracking between two dissimilar elastic layers have been extensively verified.

\section{References}

[1] Williams ML. The stresses around a fault or crack in dissimilar media. Bulletin of the Seismological Society of America 1959;49:199-204.

[2] Harvey CM, Wood JD, Wang S. Brittle interfacial cracking between two dissimilar elastic layers: Part 1-Analytical development. Composite Structures 2015, http://dx.doi.org/10.1016/j.compstruct.2015.06.080

[3] Wang S, Harvey CM. Mixed mode partition in one dimensional fracture. Journal of Key Engineering Materials 2011;462-63:616-621. Also a plenary lecture in the 8th International Conference on Fracture and Strength of Solids (FEOFS 2010), 7-9th June 2010, Kuala Lumpur, Malaysia.

[4] Harvey CM. Mixed-mode partition theories for one-dimensional fracture. PhD Thesis. March 2012, Loughborough University, UK.

[5] Wang S, Guan L. On fracture mode partition theories. Computational Material Sciences 2012;52:240-245.

[6] Wang S, Harvey CM. A theory of one-dimensional fracture. Composite Structures 2012;94:758-767. Also a plenary lecture at the 16th International Conference on Composite Structures (ICCS16), 28-30 June 2011, Porto, Portugal.

[7] Harvey CM, Wang S. Experimental assessment of mixed-mode partition theories. Composite Structures 2012;94:2057-2067. 
[8] Wang S, Harvey CM. Mixed mode partition theories for one dimensional fracture. Engineering Fracture Mechanics 2012;79:329-352.

[9] Harvey CM, Wang S. Mixed-mode partition theories for one-dimensional delamination in laminated composite beams. Engineering Fracture Mechanics 2012;96:737-759.

[10] Wang S, Harvey CM, Guan L. Partition of mixed modes in layered isotropic double cantilever beams with non-rigid cohesive interfaces. Engineering Fracture Mechanics 2013;111:1-25.

[11] Harvey CM, Wood JD, Wang S, Watson A. A novel method for the partition of mixedmode fractures in 2D elastic laminated unidirectional composite beams. Composite Structures 2014;116:589-594.

[12] Harvey CM, Eplett MR, Wang S. Experimental assessment of mixed-mode partition theories for generally laminated composite beams. Composite Structures 2015;124:10-18.

[13] Sun CT, Qian W. The use of finite extension strain energy release rates in fracture of interfacial cracks. International Journal of Solids and Structures 1997;34:2595-2609.

[14] Suo Z, Hutchinson JW. Interface crack between two elastic layers. International Journal of Fracture 1990;43:1-18.

[15] Harvey CM, Wang S. Numerical and analytical study of delamination in composite laminates. International Journal of Engineering Systems Modelling and Simulation 2012;4:120-137.

[16] Zhang Y, Wang S. Buckling, post-buckling and delamination propagation in debonded composite laminates: Part 1 Theoretical development. Composite Structures 2009;88:121130.

[17] Wang S, Zhang Y. Buckling, post-buckling and delamination propagation in debonded composite laminates: Part 2 Numerical applications. Composite Structures 2009;88:131146.

[18] Hutchinson JW, Suo Z. Mixed mode cracking in layered materials. Advances in Applied Mechanics 1991;29:63-191. 


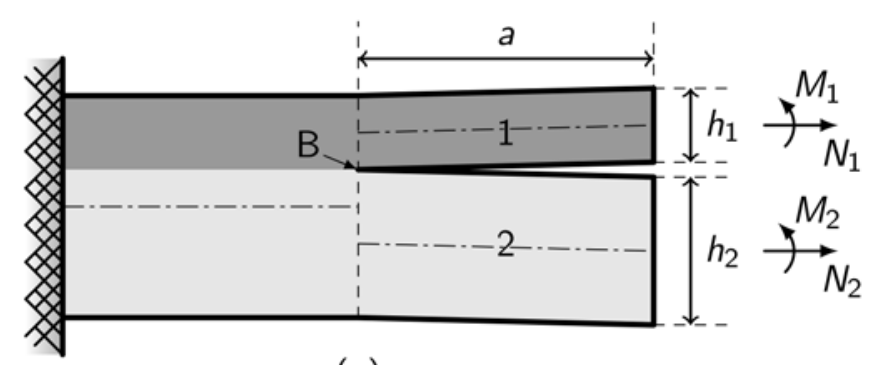

(a)

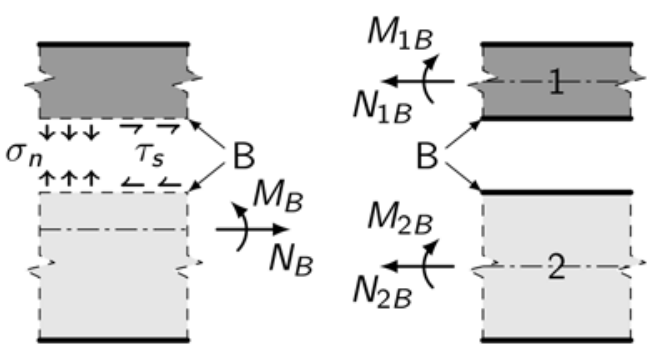

(b)

Fig. 1: A bimaterial DCB. (a) General description. (b) Interfacial stresses and crack tip forces. 


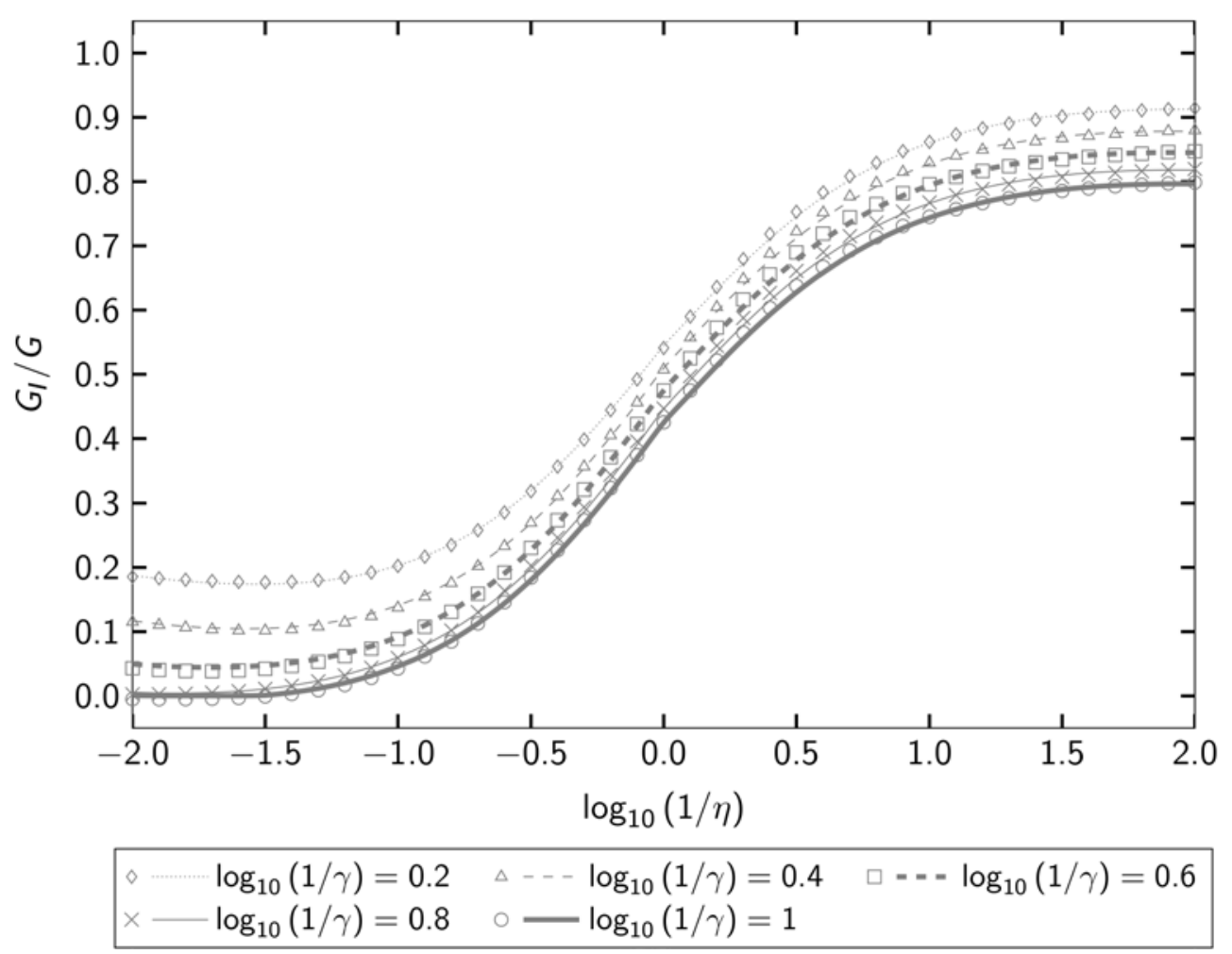

Fig. 2: Comparison of the shifting technique and FEM data for the ERR partition $G_{I} / G$ at crack extension size $\delta a=0.05$ with $M_{2 B} / M_{1 B}=0$. 


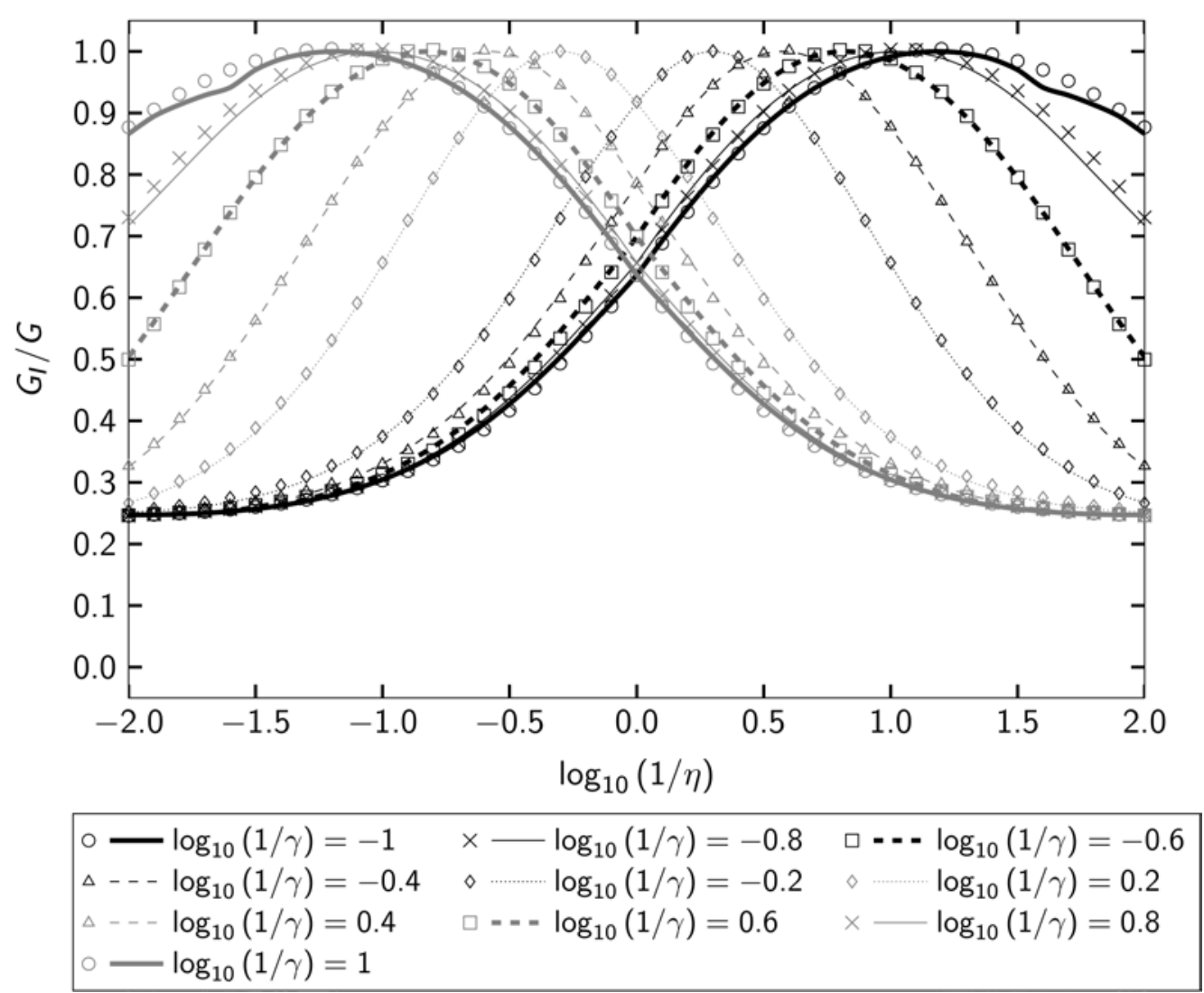

Fig. 3: Comparison of the present analytical theory and FEM data for the ERR partition $G_{I} / G$ at crack extension size $\delta a=0.05$ with $M_{2 B} / M_{1 B}=-1$. 
(a) $\delta a=0.05, M_{2 B} / M_{1 B}=0$

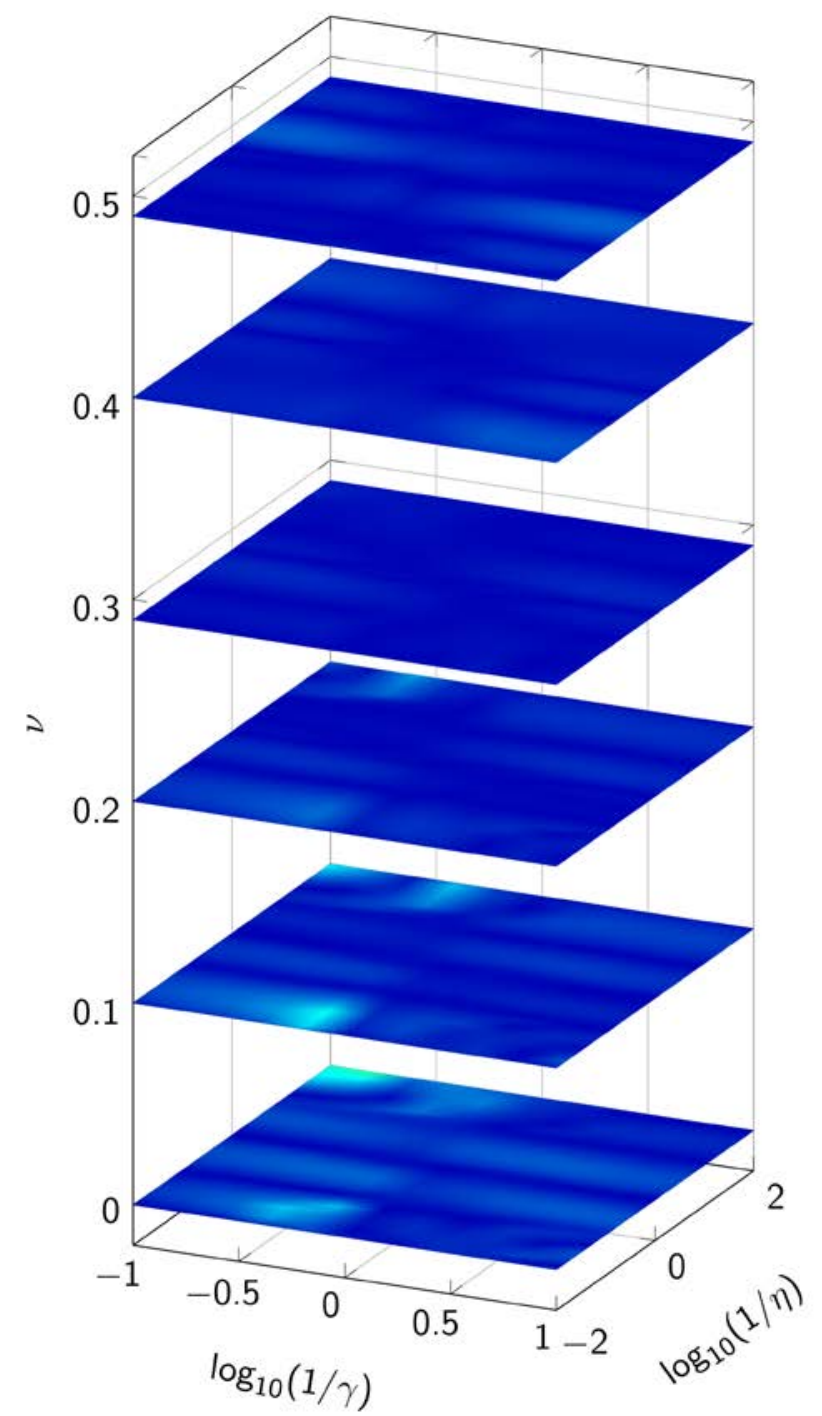

(b) $\delta a=0.05, M_{2 B} / M_{1 B}=-1$
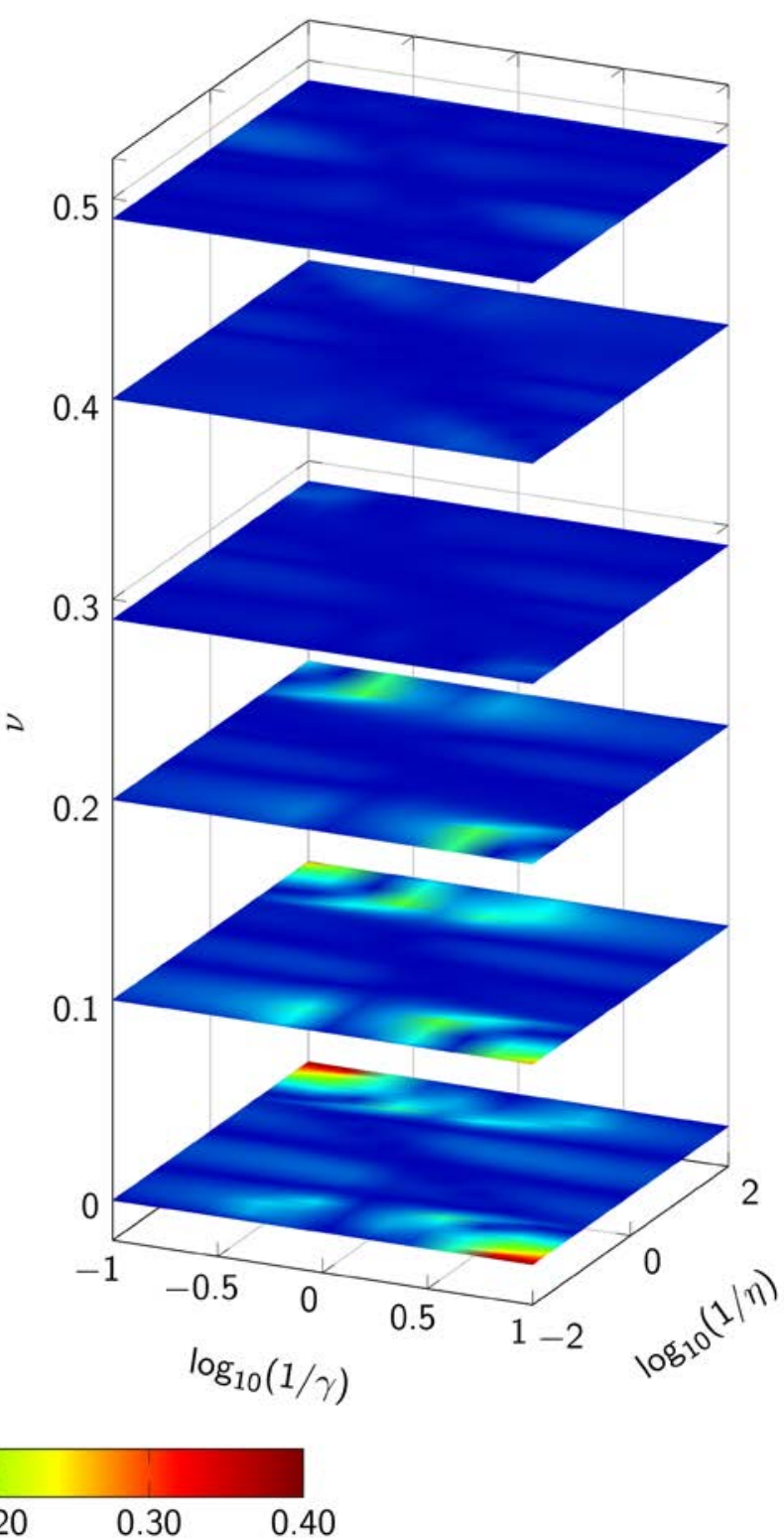

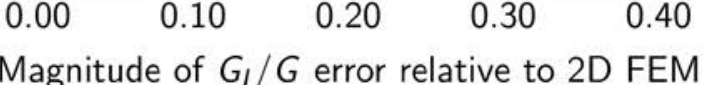

Fig. 4: Comparison of the present analytical theory and FEM data for the ERR partition $G_{I} / G$ for variable $\gamma, \eta$, and $v$ at crack extension size $\delta a=0.05$ with $M_{2 B} / M_{1 B}=0$ and $M_{2 B} / M_{1 B}=-1$. 
(a) $\delta a=0.05, M_{2 B} / M_{1 B}=0$

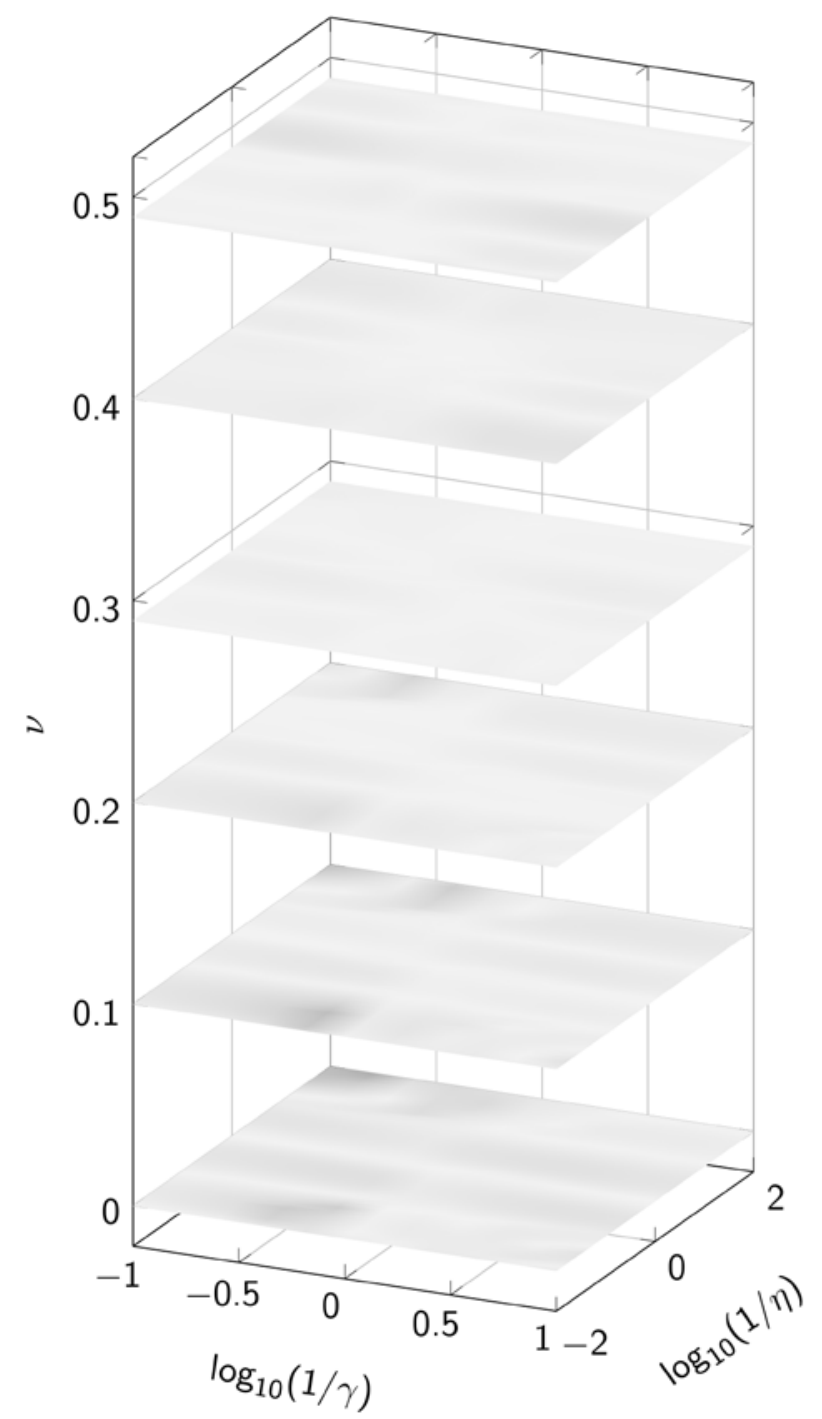

(b) $\delta a=0.05, M_{2 B} / M_{1 B}=-1$

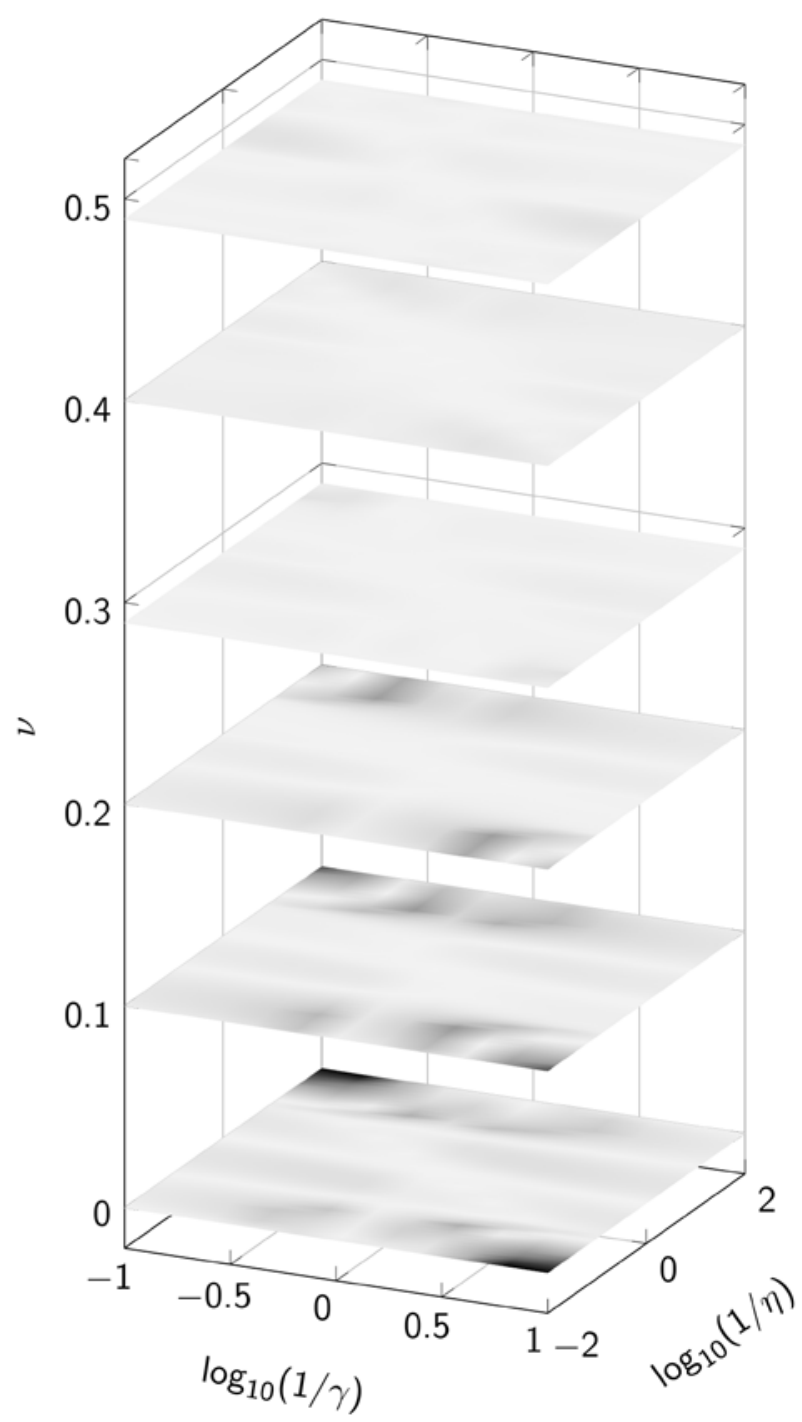

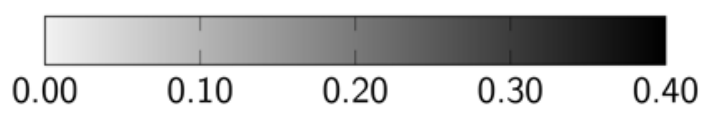

Magnitude of $G_{l} / G$ error relative to 2D FEM

Fig. 4: Comparison of the present analytical theory and FEM data for the ERR partition $G_{I} / G$ for variable $\gamma, \eta$, and $v$ at crack extension size $\delta a=0.05$ with $M_{2 B} / M_{1 B}=0$ and $M_{2 B} / M_{1 B}=-1$. 


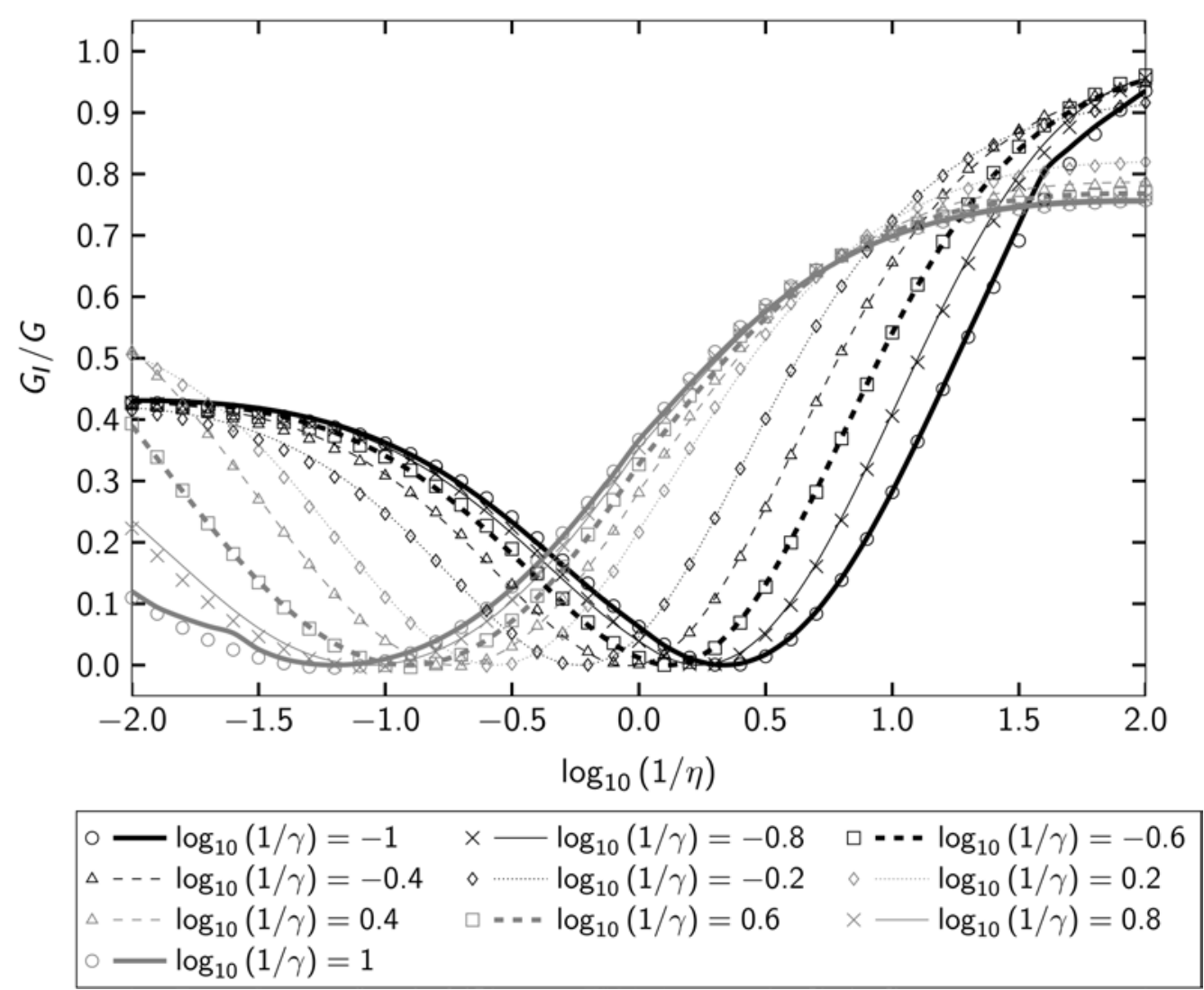

Fig. 5: Comparison of the present analytical theory and FEM data for the ERR partition $G_{I} / G$ at crack extension size $\delta a=0.05$ with $N_{1 B} / M_{1 B}=10$. 


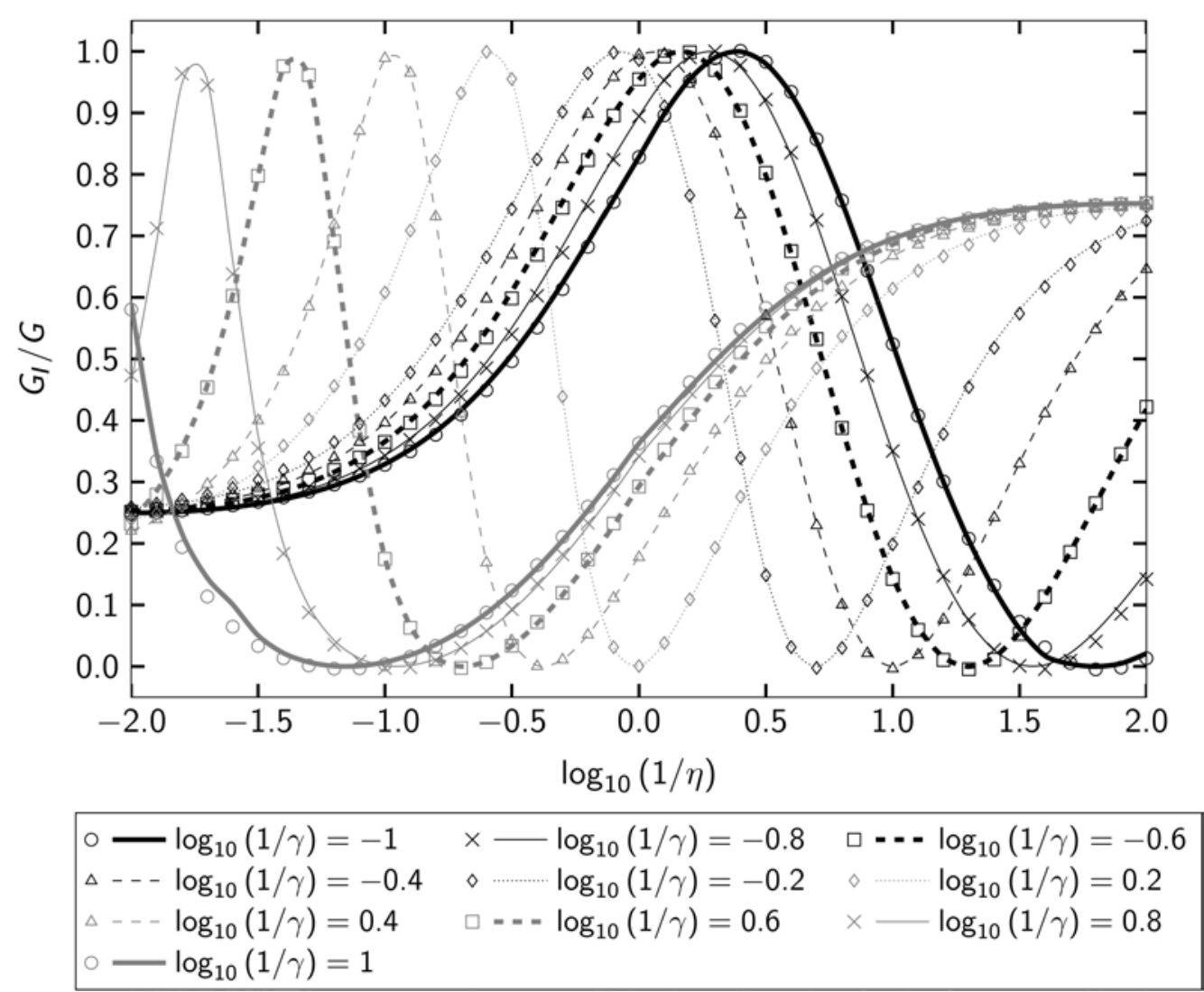

Fig. 6: Comparison of the present analytical theory and FEM data for the ERR partition $G_{I} / G$ at crack extension size $\delta a=0.05$ with $N_{2 B} / M_{1 B}=10$. 


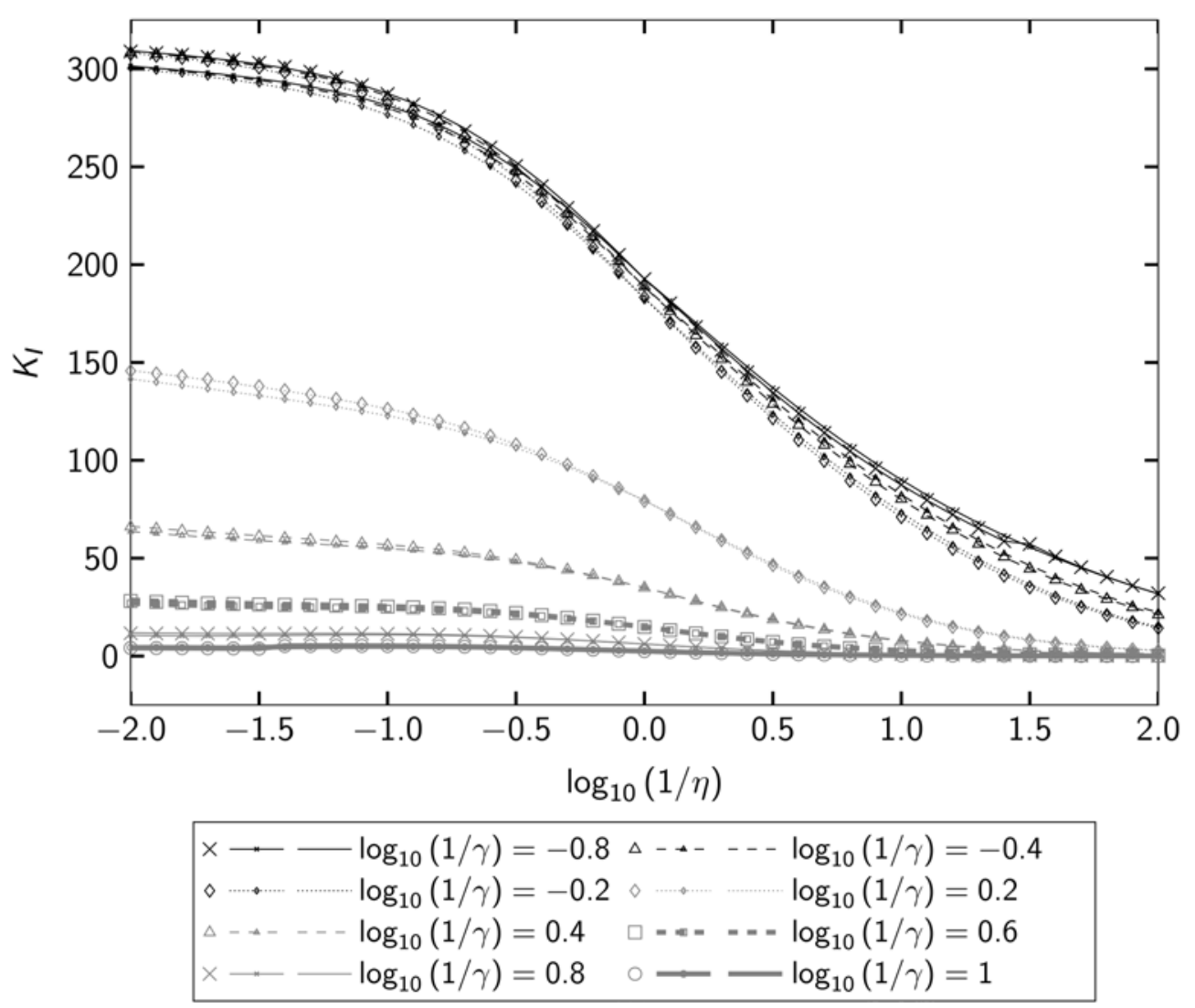

Fig. 7: Comparison of the present analytical theory and FEM data for the SIF $K_{I}$ at crack extension size $\delta a=0.05$ with $M_{2 B} / M_{1 B}=0$. 


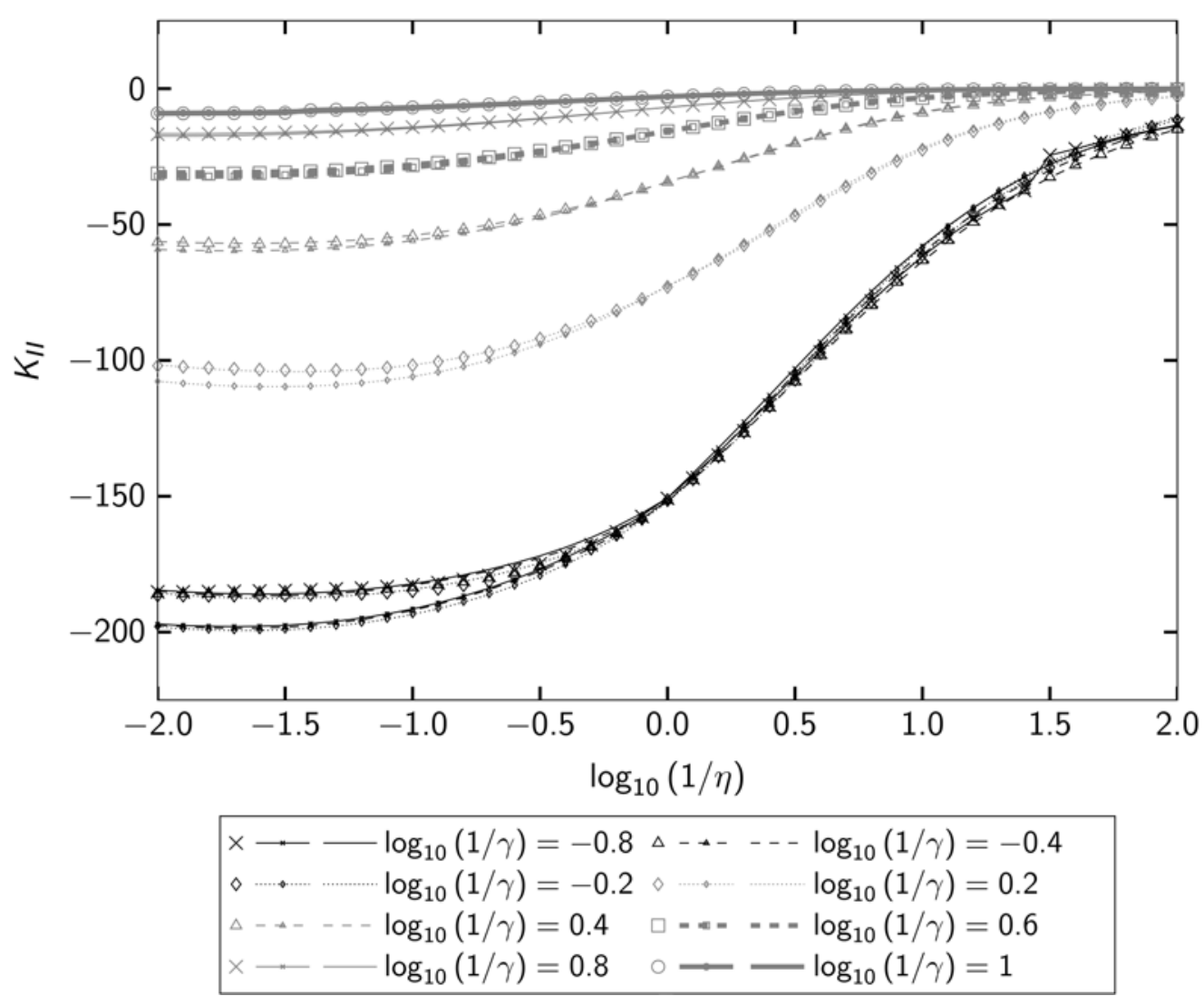

Fig. 8: Comparison of the present analytical theory and FEM data for the SIF $K_{I I}$ at crack extension size $\delta a=0.05$ with $M_{2 B} / M_{1 B}=0$. 


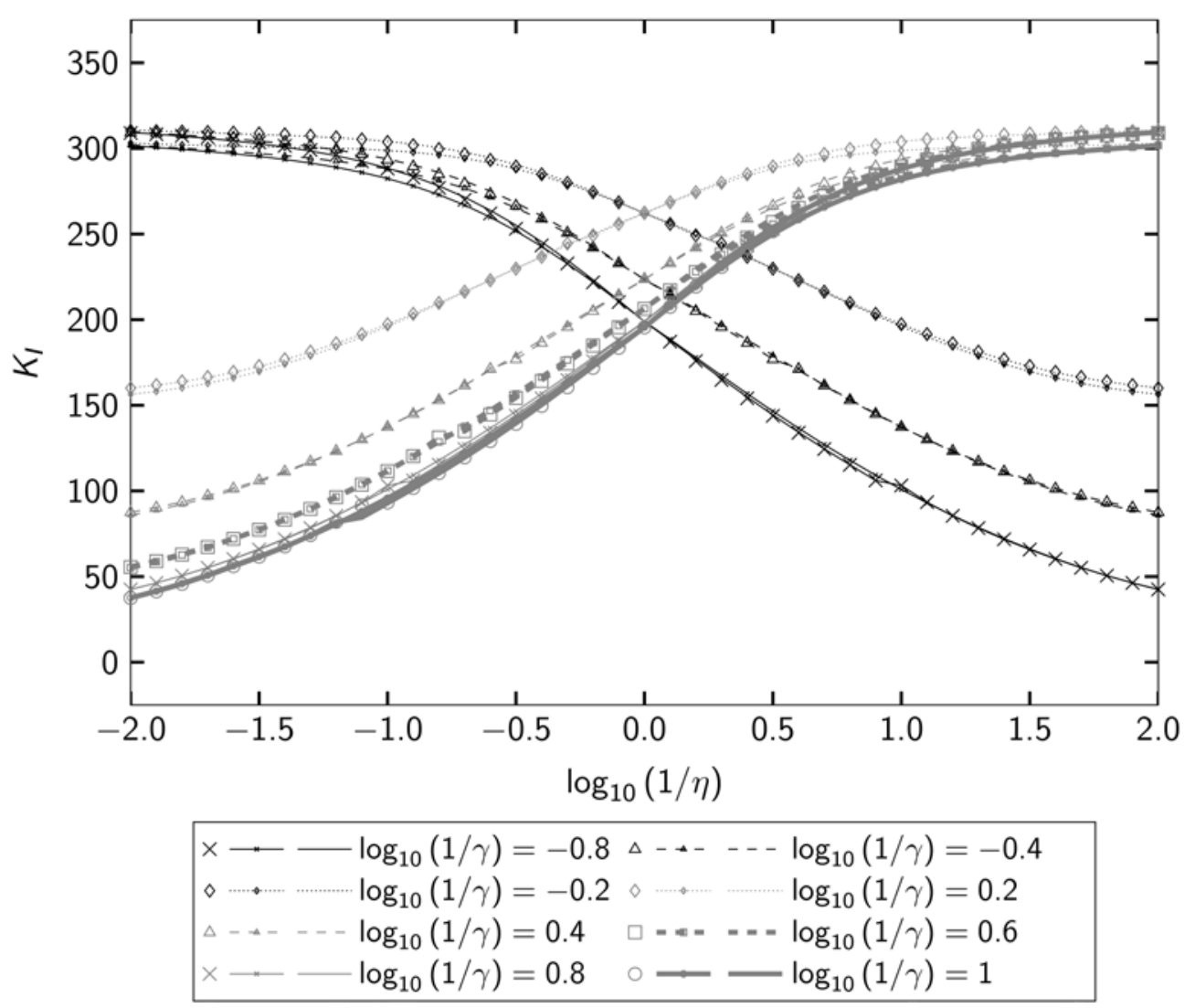

Fig. 9: Comparison of the present analytical theory and FEM data for the SIF $K_{I}$ at crack extension size $\delta a=0.05$ with $M_{2 B} / M_{1 B}=-1$. 


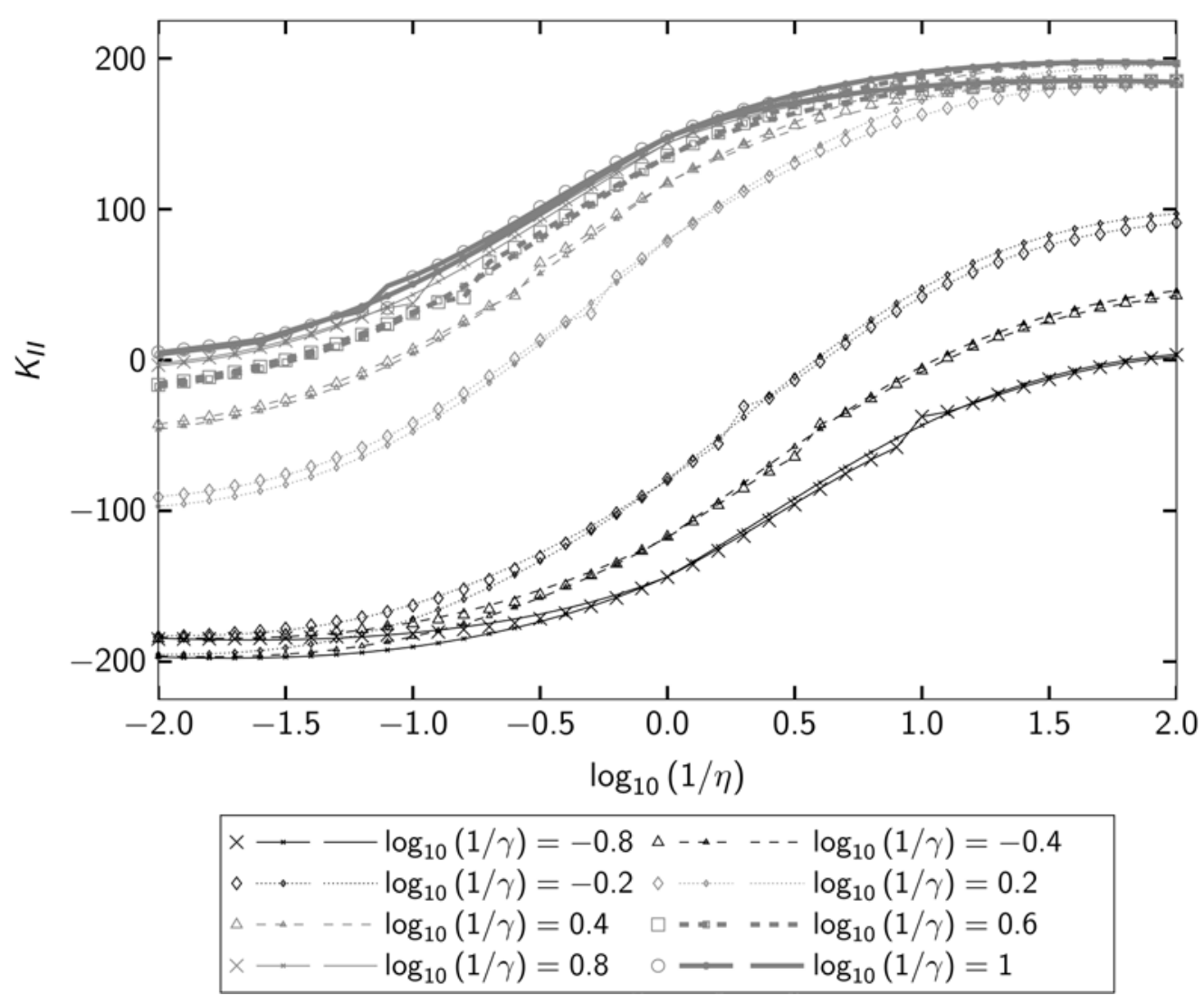

Fig. 10: Comparison of the present analytical theory and FEM data for the SIF $K_{I I}$ at crack extension size $\delta a=0.05$ with $M_{2 B} / M_{1 B}=-1$. 


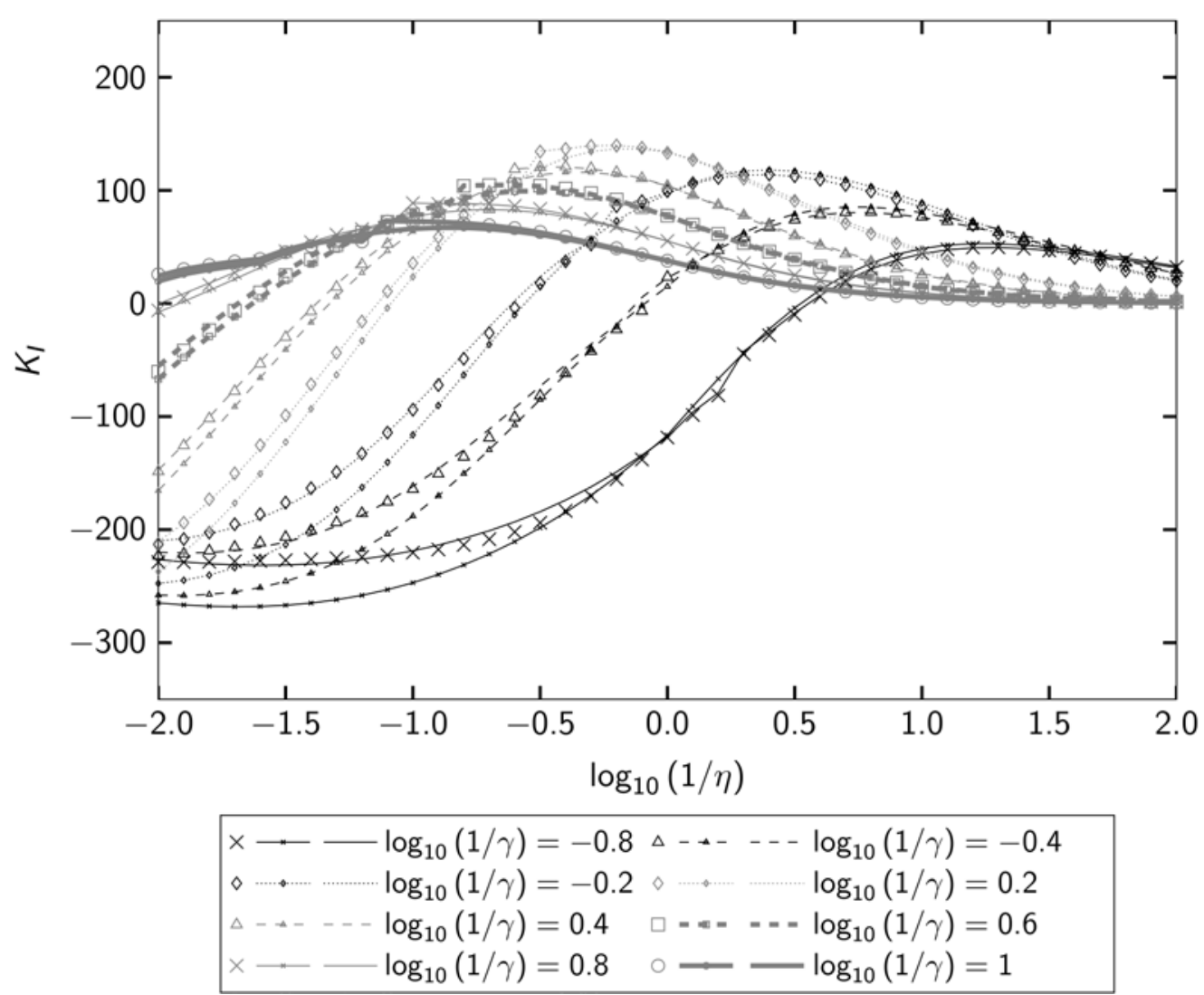

Fig. 11: Comparison of the present analytical theory and FEM data for the SIF $K_{I}$ at crack extension size $\delta a=0.05$ with $N_{1 B} / M_{1 B}=10$. 


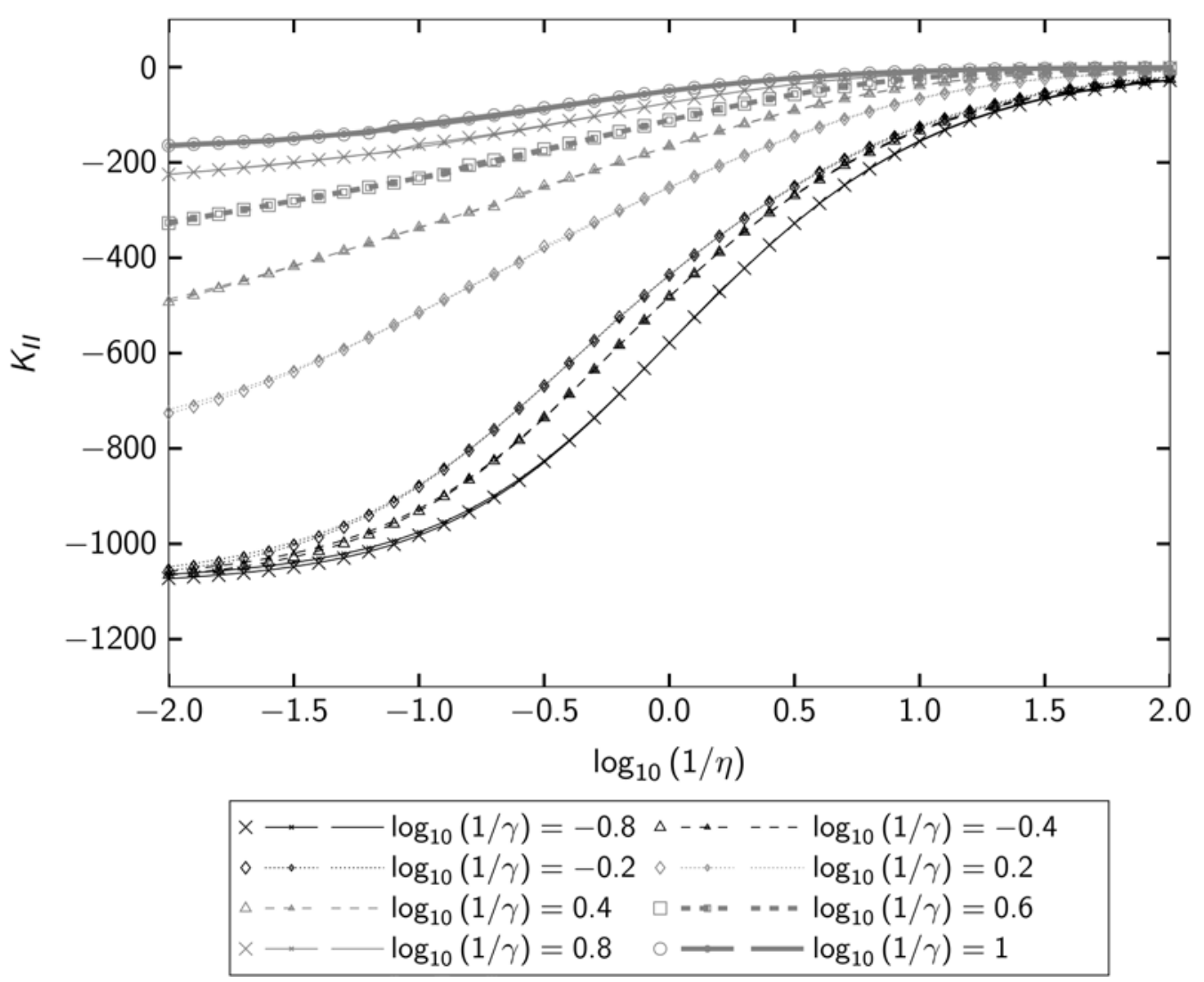

Fig. 12: Comparison of the present analytical theory and FEM data for the SIF $K_{I I}$ at crack extension size $\delta a=0.05$ with $N_{1 B} / M_{1 B}=10$. 


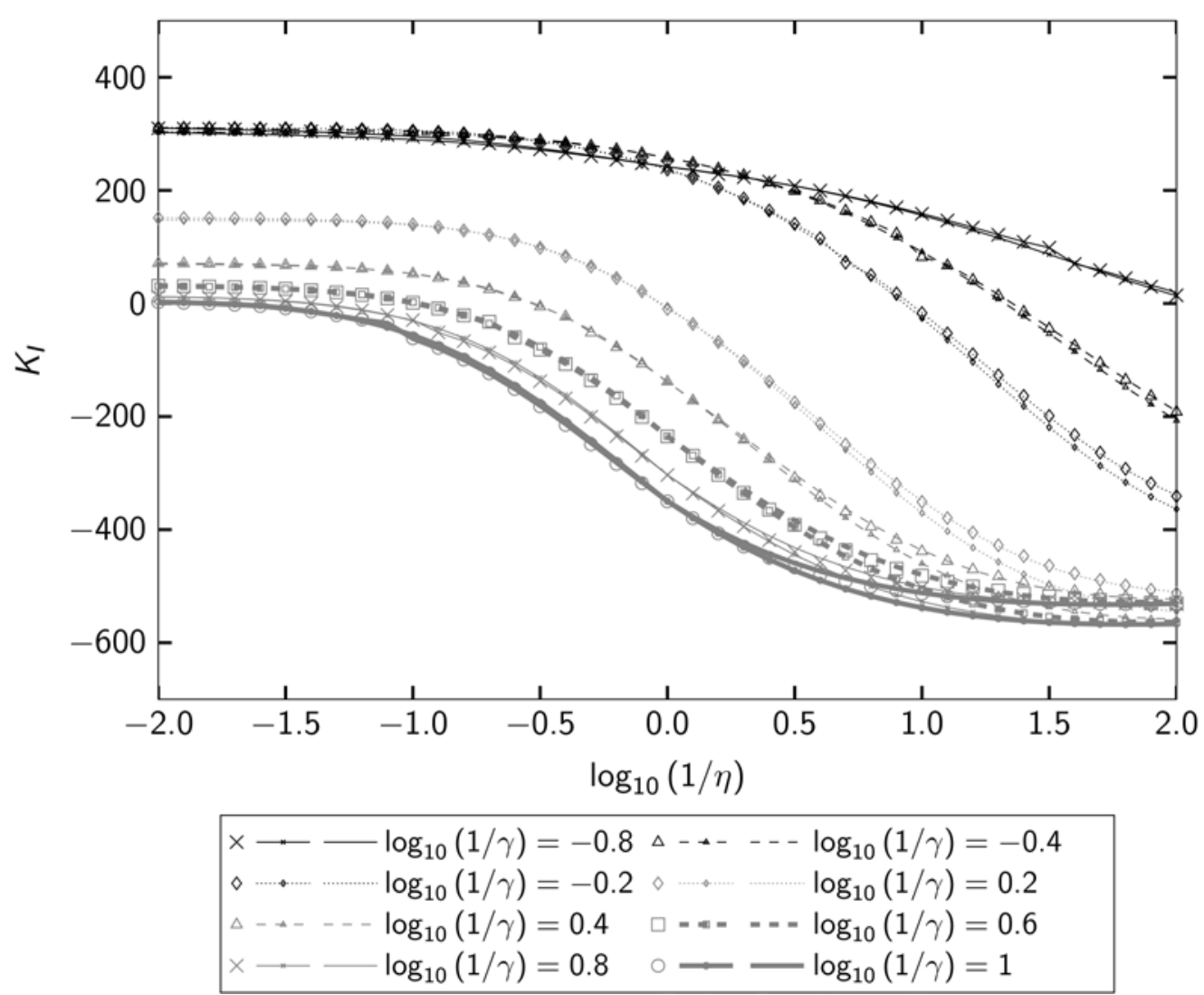

Fig. 13: Comparison of the present analytical theory and FEM data for the SIF $K_{I}$ at crack extension size $\delta a=0.05$ with $N_{2 B} / M_{1 B}=10$. 


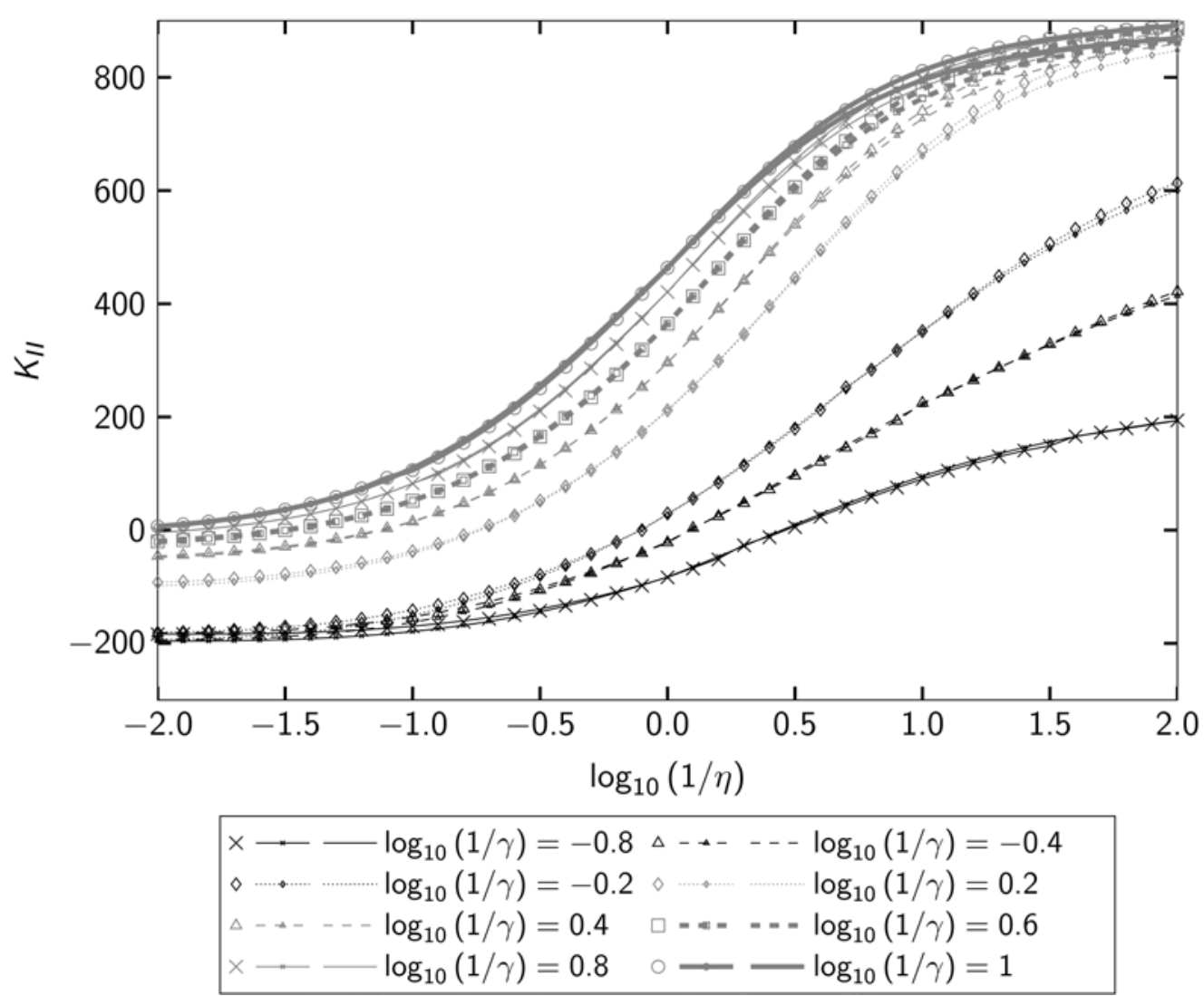

Fig. 14: Comparison of the present analytical theory and FEM data for the SIF $K_{I I}$ at crack extension size $\delta a=0.05$ with $N_{2 B} / M_{1 B}=10$. 


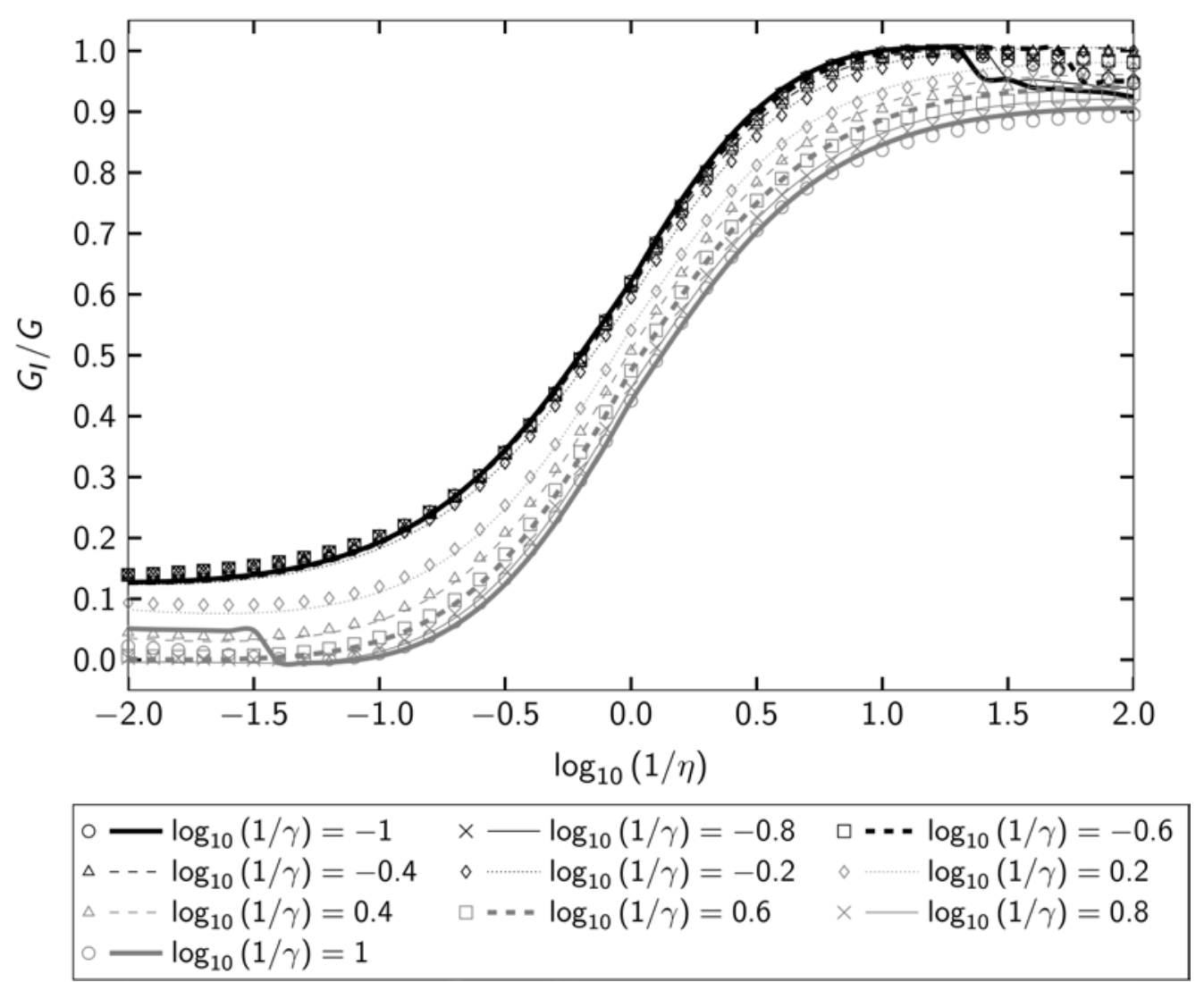

Fig. 15: Comparison of the present analytical theory and FEM data for the ERR partition $G_{I} / G$ at crack extension size $\delta a=0.01$ with $M_{2 B} / M_{1 B}=0$. 

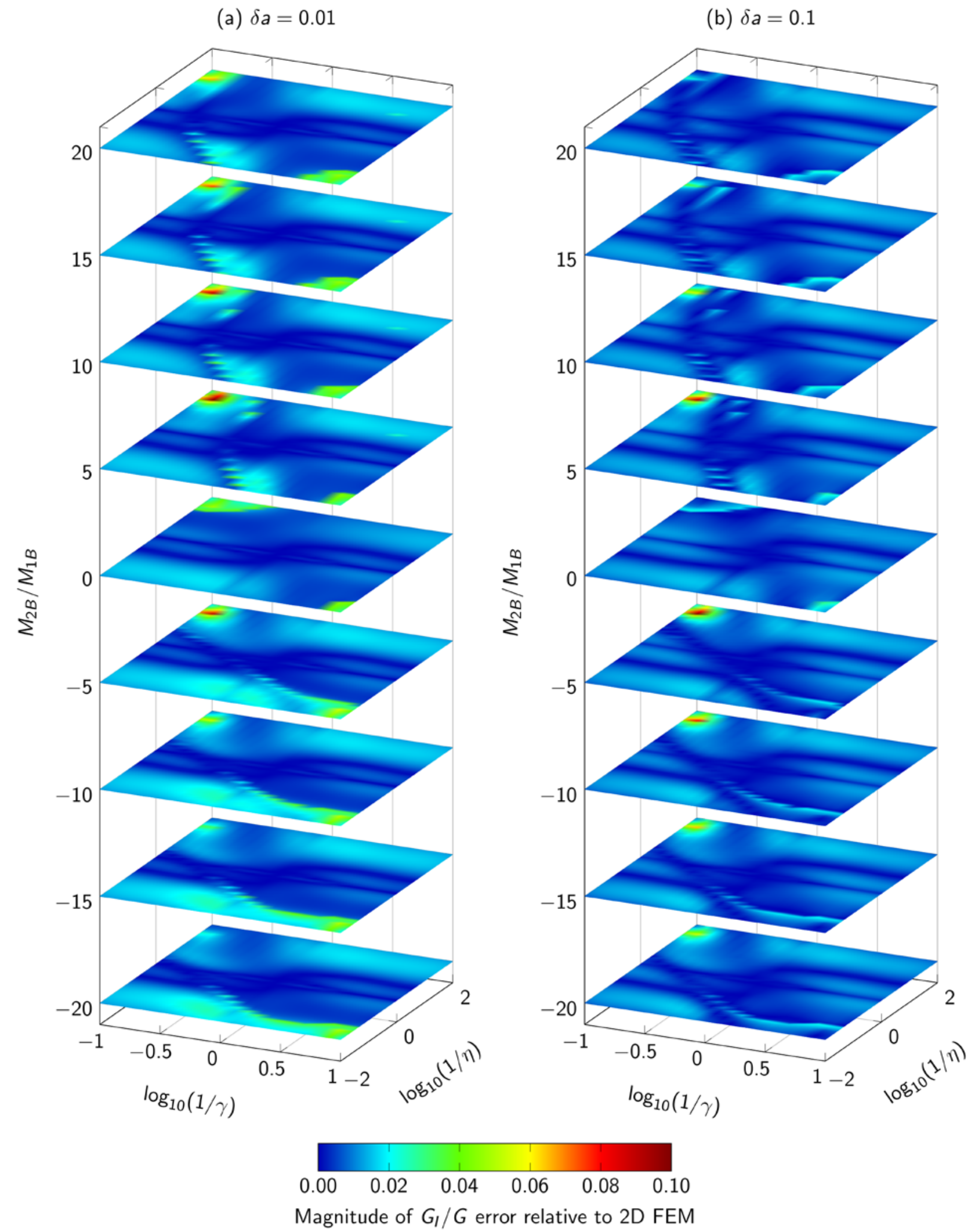

Fig. 16: Comparison of the present analytical theory and FEM data for the ERR partition $G_{I} / G$ for variable $\gamma, \eta$ and $M_{2 B} / M_{1 B}$ at crack extension sizes $\delta a=0.01$ and $\delta a=0.1$. 


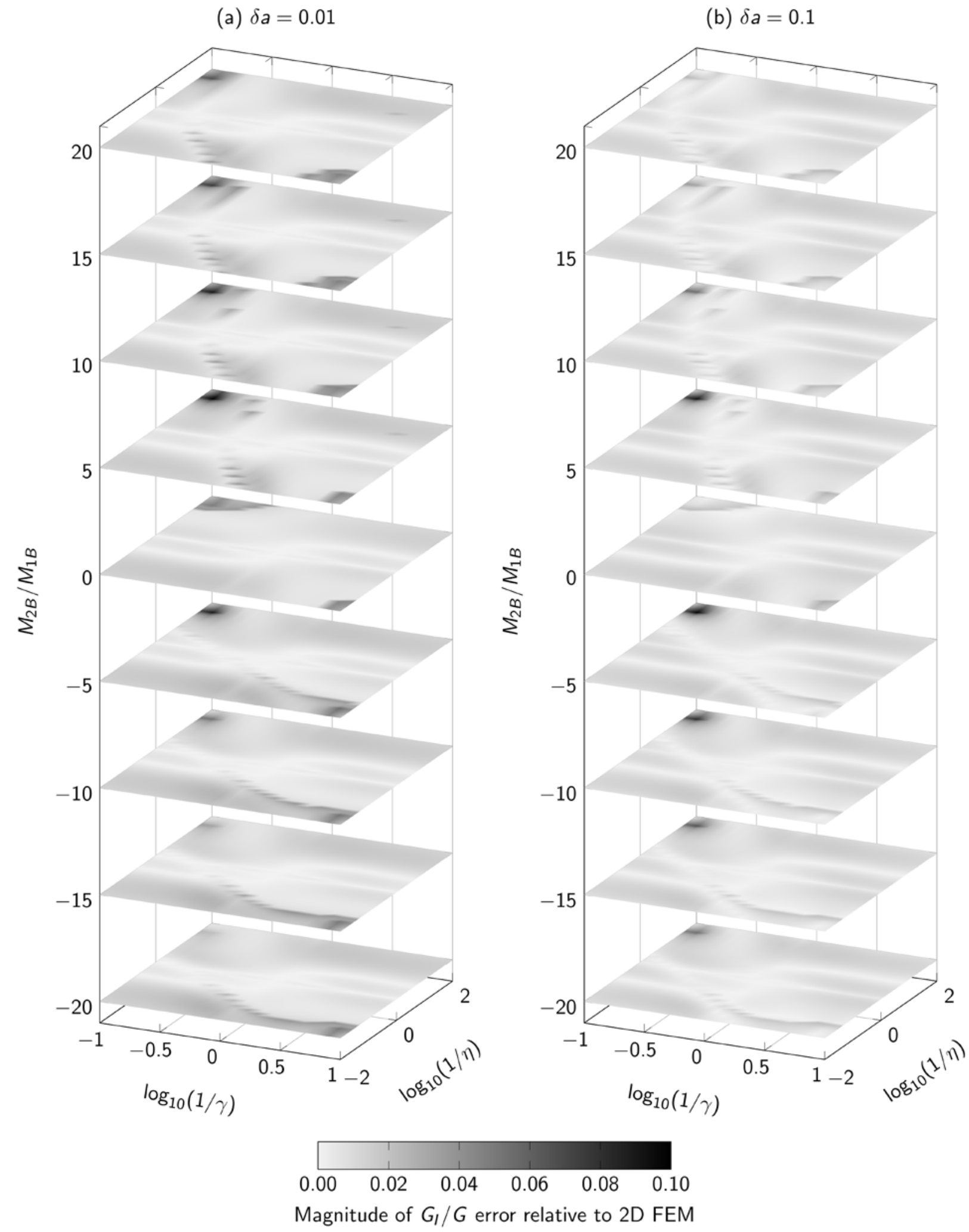

Fig. 16: Comparison of the present analytical theory and FEM data for the ERR partition $G_{I} / G$ for variable $\gamma, \eta$ and $M_{2 B} / M_{1 B}$ at crack extension sizes $\delta a=0.01$ and $\delta a=0.1$. 
(a) $\delta a=0.1$

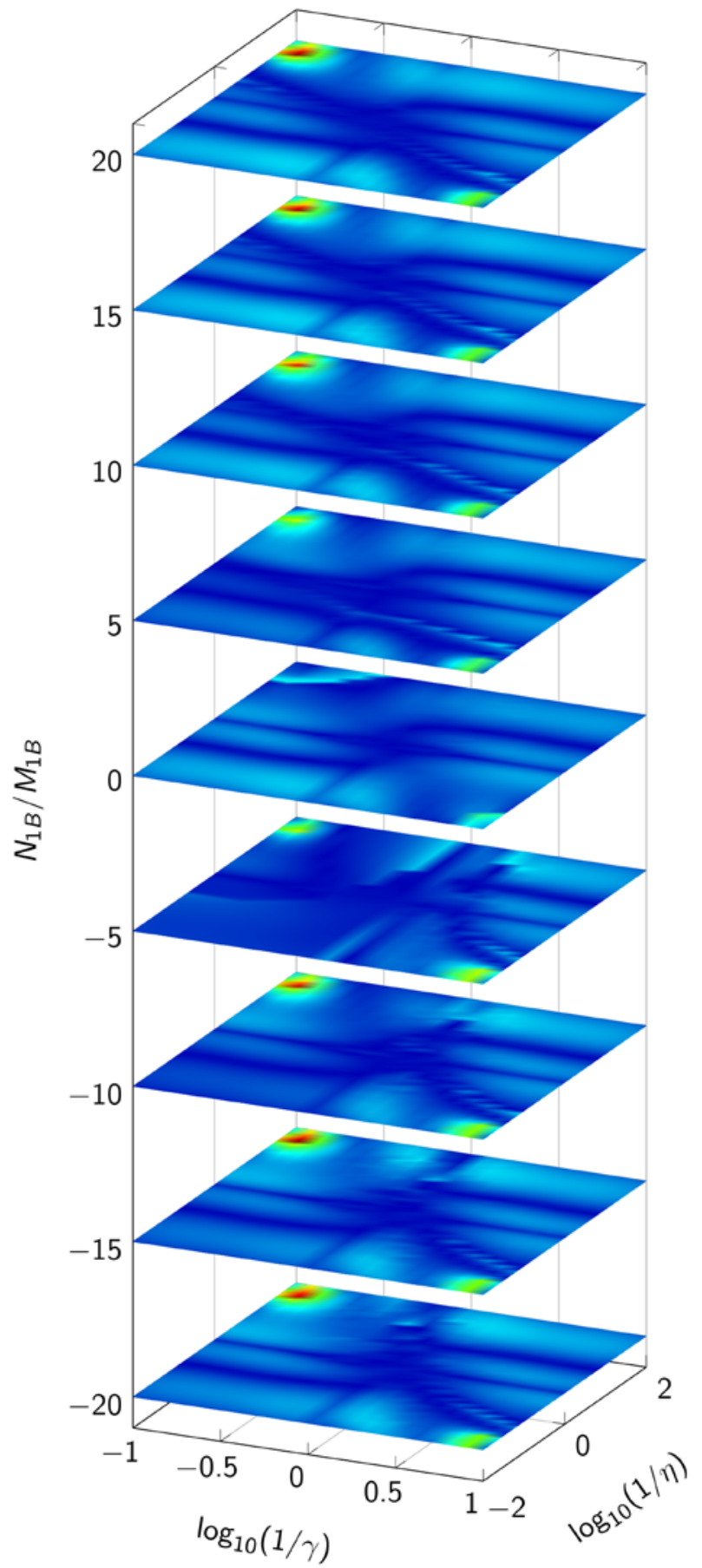

(b) $\delta a=0.1$
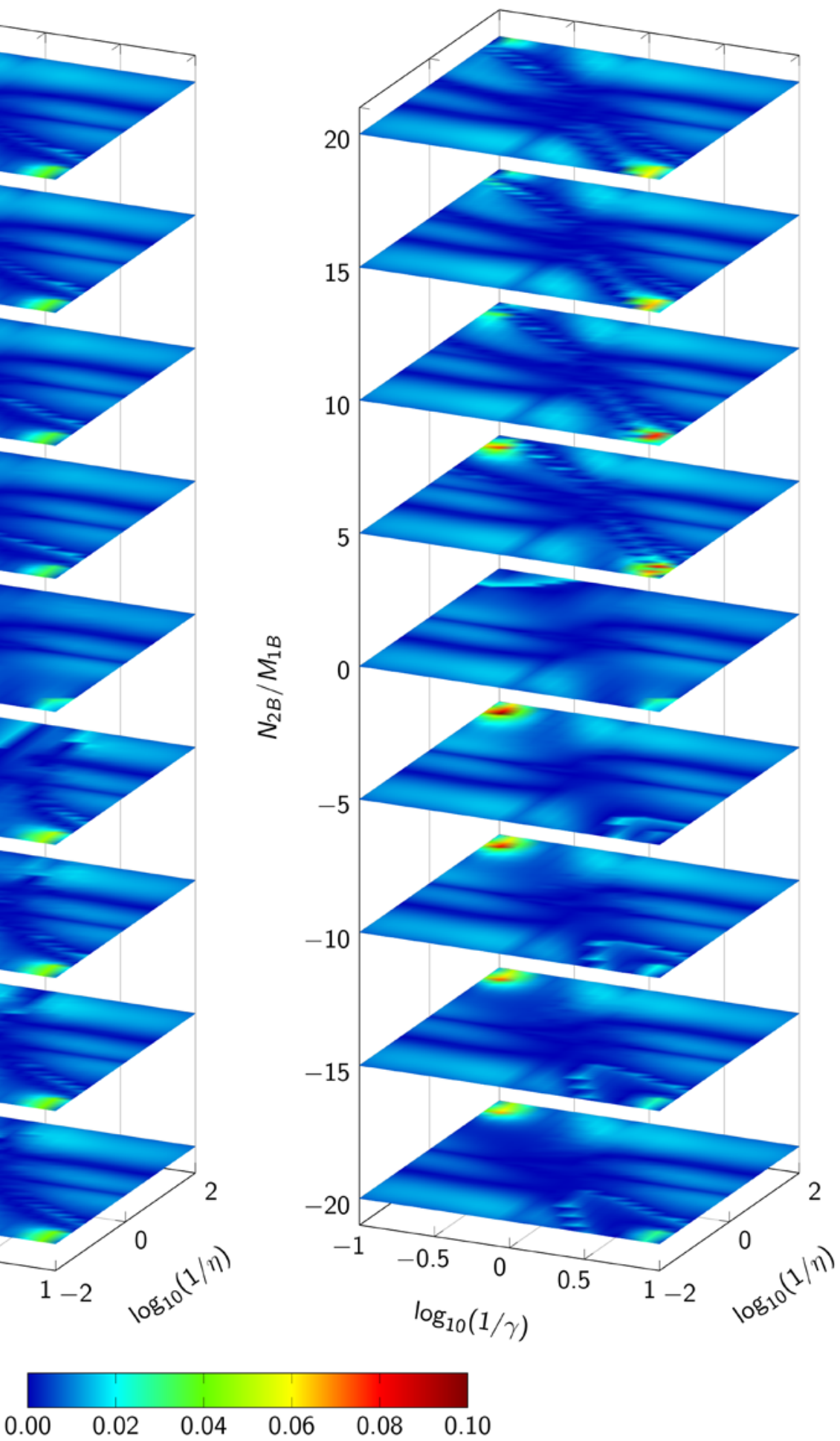

Magnitude of $G_{l} / G$ error relative to 2D FEM

Fig. 17: Comparison of the present analytical theory and FEM data for the ERR partition $G_{I} / G$ for variable $\gamma, \eta, N_{1 B} / M_{1 B}$ and $N_{2 B} / M_{1 B}$ at crack extension size $\delta a=0.1$. 

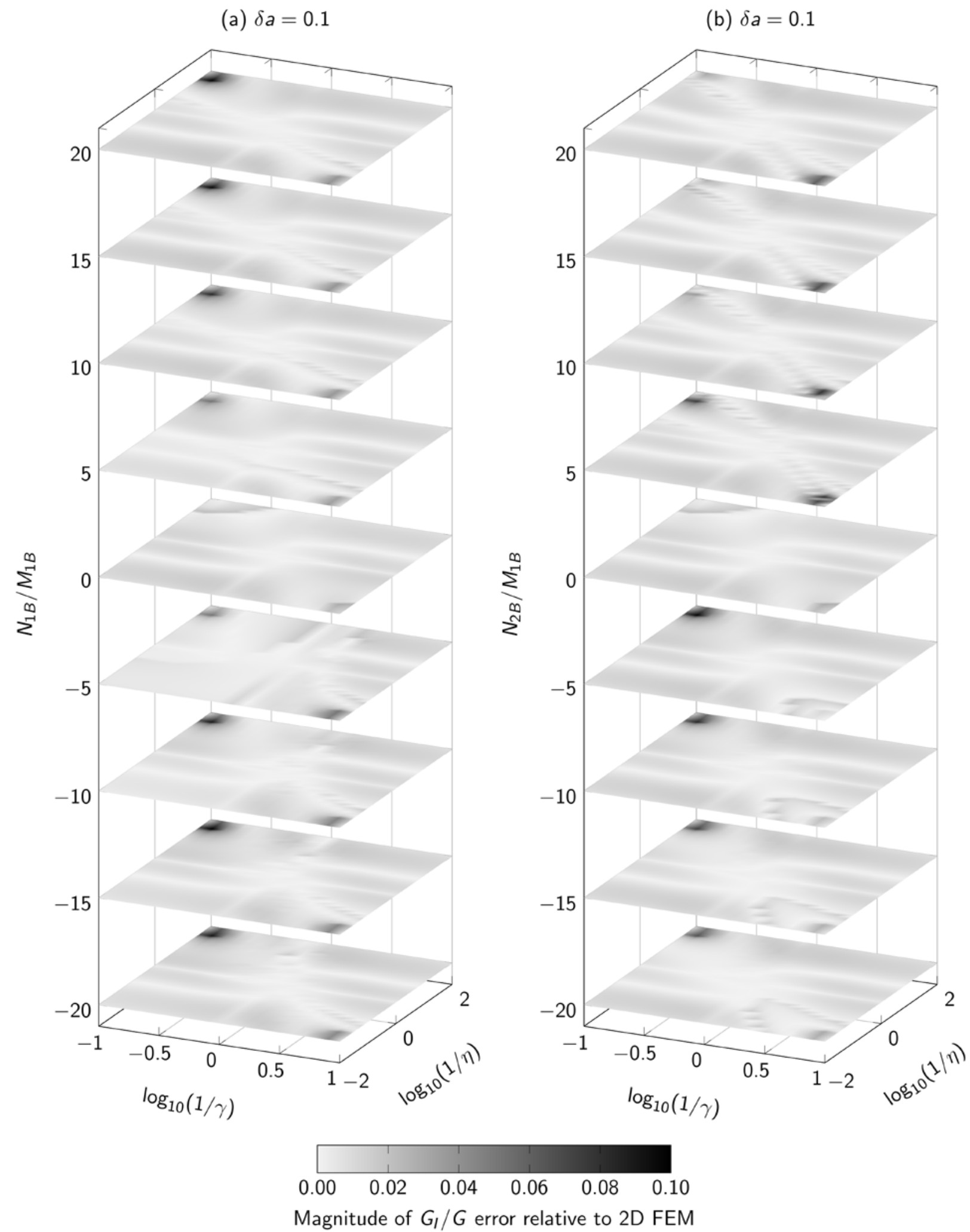

Fig. 17: Comparison of the present analytical theory and FEM data for the ERR partition $G_{I} / G$ for variable $\gamma, \eta, N_{1 B} / M_{1 B}$ and $N_{2 B} / M_{1 B}$ at crack extension size $\delta a=0.1$. 